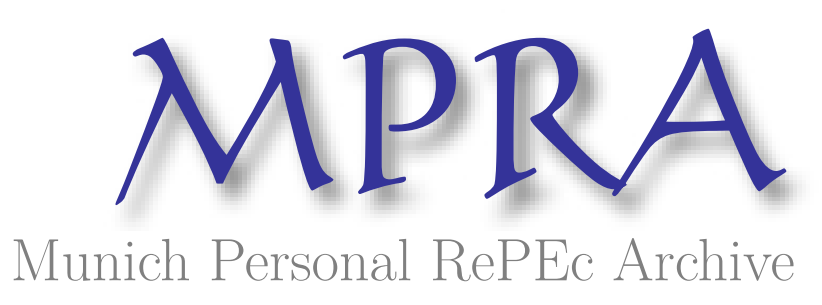

\title{
Government debt, fiscal rules and singular growth dynamics
}

Brito, Paulo

Universidade de Lisboa, ISEG and UECE

17 October 2016

Online at https://mpra.ub.uni-muenchen.de/74617/

MPRA Paper No. 74617, posted 18 Oct 2016 11:38 UTC 


\title{
Government debt, fiscal rules and singular growth dynamics
}

\author{
Paulo Brito ${ }^{\mathrm{a}}$ \\ Universidade de Lisboa, ISEG and UECE. \\ pbrito@iseg.ulisboa.pt.
}

17.10.2016*

\begin{abstract}
A fiscal rule controlling the government surplus as a function of the deviation of the actual debt ratio from a target level is introduced in an otherwise benchmark endogenous growth model in which productive government expenditures are financed by taxes and government debt. This generates a feedback mechanism from the government debt ratio to expenditure that can generate impasse-singular dynamics, in the sense that rates of growth can become locally infinitely valued. We characterize locally the different impasse-singular dynamics that can exist and discuss their consequences for the existence and characterization of general equilibrium endogenous growth paths, for different parameterizations of the fiscal rule. We present some consequences of impassesingular dynamics generated by particular fiscal rules, which are not present in regular models: existence of multiple overdeterminate balanced growth paths (BGP), existence of constraints in the domain of existence of determinate equilibrium paths converging to a regular BGP, and the existence of singular BGP's.
\end{abstract}

KEYWORDS: government debt, fiscal rules, endogenous growth, impasse-singular dynamics.

JEL CODES: C61, E62, H63

\footnotetext{
*I acknowledge helpful comments by Emmanuelle Augeraud-Veron and Alfred Greiner on previous versions of this paper. It was presented at the Conference "Financial and Real Interdependencies: volatility , inequalities and economic policies", Lisbon, May 30 2015, Católica Lisbon School of Business and Economics and at the 11th Annual Meeting of the Portuguese Economic Journal, Coimbra, July 2, 2016, Faculty of Economics University of Coimbra. Financial support from national funds by FCT is acknowledged. This article is part of the Strategic Project UID/ECO/00436/2013
} 


\section{Introduction}

Within different contexts, and with several justifications, there is a widespread adoption of fiscal rules (see Kinda et al. (2013) for a survey) introducing targets on the primary surplus, on the government debt relative to GDP, or both. Rules can be explicit or implicit. A clear example of the first type are the rules agreed upon within the Treaty on Stability, Coordination and Governance in the Economic and Monetary Union, in the chapter known as the Fiscal Compact, that bind the fiscal policy of most members of the European Union. There is also empirical evidence that there are fiscal reaction functions relating the primary surplus to the level of debt, even for countries as the U.S whose fiscal policy is not bound by international treaties (see Bohn $(1998,2008)$ and Ghosh et al. (2013)).

Budget rules, both in the form of a budget balance or of any other form of fiscal target, endogenize one or more fiscal policy instruments and introduce a feedback mechanism relating government expenditures or taxes to the level of government debt.

In the macroeconomic theory literature the case which has been more studied is related to the consequences of budget balance rules over transition dynamics. Papers in this strand of literature (see Schmitt-Grohe and Uribe (1997), or a recent contribution Nishimura et al. (2015)), find that a budget balance rule can be destabilizing and a source of (permanent) indeterminacy. In all those papers the governement deficit is potentially financed by taxes only. A budget rule introduces a pro-cyclical mechanism, through the effect of endogenously set taxes distorting the rate of return of capital, which can generate a self-fulfilling future change in taxes.

In that strand of models the government expenditures are not productive, there is not economic growth, and the government debt dynamics is absent. Since Arrow and Kurz (1970) it is know that public capital is a relevant source of growth. In most actual economies the government can be seen as a productive sector specializing in the provision of goods and services which have an intrinsic aggregate nature. It is, therefore, a generator of externalities, and consequently it is a major source of economic growth. In the endogenous growth literature Barro (1990) offers a benchmark model in which the government expenditures are productive ( see Irmen and Kuehnel (2009) for a survey on the voluminous literature following that paper). However, government financing, by taxes or debt, has also an aggregate effect over the cost of capital that can partially or totally offset the positive effect of externalities' production. Fiscal rules introduce another transmission mechanism for fiscal policy which tends to work through the expectations of future changes in the rate of return of the economy.

Contributions dealing with productive government expenditures, economic growth, debt financing and fiscal rules can be separated into two strands, depending on the nature of the fiscal rule: Futagami et al. (2008) consider an adjustment equation where the variation of the debt over capital ratio is adjusted against a benchmark while Greiner (2008), Kamiguchi and Tamai (2012) and (Greiner and Fincke, 2015, p. 169-173) consider a rule in which 
the primary surplus is adjusted to the actual ratio of government debt over GDP relative to a target. This literature has two shortcomings: either the rules are unrealistic (they feature simply a feedback mechanism for government debt), or the dynamic implications of the feedback mechanism introduced by the rules were not fully addressed. Indeed, in some of these papers (for instance, in Greiner and Fincke (2015)) singular dynamics, taking the form of impasse-singularities, is present.

In this paper we extend an otherwise benchmark Barro (1990) model, in which government expenditures are productive and are financed by distortionary taxes and government debt, by introducing a (symmetric) fiscal rule according to which the primary surplus is a linear function of the deviation of the debt to GDP ratio relative to a target level (as in Bohn (1998) and Greiner and Fincke (2015) ). We are not concerned with the determination or the setting of an optimal target but with the existence and characterization of the impasse-singularities that different fiscal rules can generate.

A general analysis for two-dimensional dynamic general equilibrium (DGE) models in the presence of impasse-singularities is provided in Brito et al. (2017a). In that paper it is shown that the vector field in the dynamic system representing the DGE is non-Lipschitzian which implies that the existence of solutions for all $t \in[0, \infty)$, for any given initial value for the non-anticipating variables, is not guaranteed. This means that the existence of DGE paths should be explicitly checked before any attempt to characterize them. There are several symptoms of the presence of impasse-singularities: existence of infinitely-valued eigenvalues of the jacobian of the dynamic system, multiplicity of steady-states with the same type of local dynamics (e.g. two saddle-points as in Greiner and Fincke (2015)), or the existence of changes in the determinacy properties of the DGE paths along the transition path (if it exists). In the presence of impasse-singularities the local analysis in the neighborhood of a steady-state can be misleading. In Brito et al. (2017a) it is proved that if there is a unique DGE path, and there is an impasse-singularity, only four cases can happen: black-hole singularities and no transitional dynamics, white-hole singularities and transitional dynamics confined to a subset of the state space, or worm-hole dynamics and trajectories cross the impasse surface but change their determinacy properties, from locally determinate to locally indeterminate, or from locally indeterminate to locally determinate.

In this paper we use the method presented in Brito et al. (2017a) ${ }^{1}$ and go beyond by extending it along three dimensions: first, we deal with an endogenous growth model in which the transition dynamics can exist along a BGP and the steady states refer to the detrended variables; second, we have multiplicity of steady states; and, third, we also have multiple impasse-singularities. In Brito et al. (2017a) the steady state refers to a stationary DGE path, and there is at most one isolated steady state and one isolated impasse-singularity. The method consists in studying local dynamics not only in the neighborhood of the steady

\footnotetext{
${ }^{1}$ In both papers we deal with implicit differential equations by using the approach and terminology presented in Zhitomirskii (1993) and Llibre et al. (2002) and extending to cases in which there are steady states. See also Riaza (2008).
} 
state but also in the neighborhood of the impasse-singularity. However, as we proved in that paper, those two types of local analysis although providing necessary conditions for the existence and the characterization of DGE paths are not sufficient: there are existence conditions of a global nature and a complete characterization of the dynamics, in particular the existence of temporary indeterminacy, also depends on global type of conditions.

We present a thorough local bifurcation analysis in the space of two fiscal rule parameters: the debt target and the speed of adjustment to that target. We then select some specific parameter values, and present the global phase diagrams for cases that cannot occur in regular DGE models, in which there are multiple over-determinate BGP's, or there are bounded transitional determinate DGE dynamics converging to a regular BGP path, or to singular BGP path.

The rest of the paper is organized in the following way: in section 2 we present the model, in section 3 we characterize analytically local dynamics both at the impasse surface and at the BGP, section 4 presents the phase diagrams for a sample of possible DGE scenarios, and section 5 closes the paper ${ }^{2}$.

\section{The model}

The representative private consumer-producer maximizes the intertemporal utility functional

$$
\int_{0}^{\infty} \frac{C(t)^{1-\sigma}}{1-\sigma} e^{-\rho t} d t
$$

where $C$ is consumption subject to the budget constraint

$$
\dot{K}+\dot{B}=(1-\tau(t))(r(t) B(t)+Y(t))-C(t),
$$

where $K$ and $B$ are the stocks of physical capital and government debt, $\tau$ is the income-tax rate, $r$ is the rate of interest on government bonds, and $Y$ is the income from production ${ }^{3}$. We assume that the rate of time preference, $\rho$, is positive, and the intertemporal elasticity of substitution is below unity, $\sigma \geq 1$. The initial levels for the two stocks are given and are non-negative in present value terms $\lim _{t \rightarrow \infty}(K(t)+B(t)) e^{-\int_{0}^{t} r(s) d s} \geq 0$.

There is only one good in this economy, which is produced by a technology using public goods provided by the government. We posit a Cobb-Douglas production function

$$
Y(t)=K(t)^{\alpha} G(t)^{1-\alpha}
$$

\footnotetext{
${ }^{2}$ The proofs are assembled at the appendix.

${ }^{3}$ (Greiner and Fincke, 2015, p. 169-173) assume that only the income generated by private sources is taxed. That is, there are no taxes over the return on the private investment on government bonds. This assumption changes significantly the types of dynamics that can occur.
} 
where $G$ is the government expenditure, and $\alpha \in(0,1)$ is the income share of private capital. A non-arbitrage condition holds such that the gross rate of interest on government bonds is instantaneously equalized to the marginal productivity of capital,

$$
r(t)=\alpha K(t)^{\alpha-1} G(t)^{\alpha-1} .
$$

From household optimality conditions we derive the Euler equation

$$
\frac{\dot{C}}{C(t)}=\frac{(1-\tau(t)) r(t)-\rho}{\sigma}
$$

and the transversality condition $\lim _{t \rightarrow \infty} C(t)^{-\sigma}(K(t)+B(t)) e^{-\rho t}=0$.

The government finances expenditures by taxing all types of income and issuing bonds such that the budget constraint

$$
\dot{B}=r(t) B(t)-S(t)
$$

holds, where $B$ is the stock of outstanding government bonds (indeed debt) and the primary surplus is

$$
S(t)=\tau(t)(r(t) B(t)+Y(t))-G(t) .
$$

We consider the fiscal rule similar to the one introduced in Greiner (2008), Kamiguchi and Tamai (2012) and (Greiner and Fincke, 2015, p. 169-173),

$$
S(t)=\phi(B(t)-\bar{B}(t))
$$

where $\phi$ parameterizes the speed of the adjustment of the actual level of debt to a timevarying target $\bar{B}(t)$. We assume that the debt target is set such that its ratio with the GDP is constant

$$
\frac{\bar{B}(t)}{Y(t)}=\bar{b}, \text { for all } t \in[0, \infty) .
$$

We assume both parameters defining the policy, $\phi$ and $\bar{b}$, are strictly positive. A higher $\phi$ speeds up the adjustment of the actual level of the government debt to the target. We also assume that the tax rate is fixed parametrically and that the government implements the rule by controlling government expenditures.

Consolidating the government and the household budget constraints we get the good's market clearing equation

$$
\dot{K}=Y(t)-C(t)-G(t) .
$$

In order to render them clear we formally define both dynamic general equilibrium (DGE) and equilibrium balanced growth (BGP) paths:

Definition 1 (DGE). A DGE is a trajectory $(K(t), C(t), B(t), G(t), r(t))_{t \in[0, \infty)}$ satisfying, for all $t \in[0, \infty)$, equations (2), (6) and (3) together with the fiscal rule (5), the transversality and initial conditions, $K(0)=K_{0}$ and $B(0)=B_{0}$, where $K_{0}$ and $B_{0}$ are given. 
Definition 2 (BGP). A BGP path is a particular DGE path such that the rates of growth for $K, C, B$ and $G$ are constant and $r$ is constant.

In the rest of the paper we denote the ratios of government debt and government expenditures over the GDP by $b \equiv B / Y$ and $g \equiv G / Y$, respectively.

Setting $G=g Y$ in the production function we can rewrite it as a linear function of the capital stock $Y=A(g) K$, where $A(g) \equiv g^{\frac{1-\alpha}{\alpha}}$. Therefore, the average productivity of capital is an increasing function of $g$ and its curvature depends on $\alpha$. By assuming $\alpha>1 / 2$ we set it as concave.

This implies that the interest rate, $r=r(g)=\alpha A(g)$, is also an increasing (and concave if $\alpha>1 / 2$ ) function of the ratio of government expenditures over the GDP. In addition, the rate of growth of consumption is also an increasing (and concave) function of the government expenditure over the GDP

$$
\frac{\dot{C}}{C}=\gamma(g) \equiv \frac{(1-\tau) r(g)-\rho}{\sigma} .
$$

\subsection{Endogenous debt adjustment}

If we eliminate the budget surplus, $S$, in equations (4) and (5) we get a relationship between the government debt and the government expenditure, engineered by the fiscal rule, $\tau(t)(1+$ $r(t) b(t))-g(t)=\phi(b(t)-\bar{b})$. As the debt ratio and the government expenditures are both endogenous, the government has one degree of freedom consisting in fixing the income tax rate exogenously. From now on we assume that it is a constant parameter: $\tau(t)=\tau$.

In order to get explicit expressions, it is convenient to express the endogeneity of the expenditure ratio regarding the debt ratio, introduced by the fiscal rule and the exogeneity of the tax rate, by the inverse relationship

$$
b(t)=b(g(t)) \equiv \frac{g(t)-\bar{g}}{\tau r(g(t))-\phi} .
$$

In equation (8) we introduce two critical levels for the expenditure ratio: the level associated with zero government debt,

$$
\bar{g} \equiv \tau+\phi \bar{b},
$$

and the level associated with an unbounded level for $b$,

$$
\tilde{g} \equiv\left(\frac{\phi}{\alpha \tau}\right)^{\frac{\alpha}{1-\alpha}},
$$

which is attained when the tax collected from the interest on government bonds is equal to the rate of adjustment of the debt ratio, $\tilde{g} \equiv\{g: \operatorname{\tau r}(g)=\phi\}$. The relationship of the 
expenditure ratio relative to those two critical values determines the creditor position of the government: if $\min \{\bar{g}, \tilde{g}\}<g<\max \{\bar{g}, \tilde{g}\}$ then, because $b(g)<0$, the government is a creditor; if $g<\min \{\bar{g}, \tilde{g}\}$ or $g>\max \{\bar{g}, \tilde{g}\}$ then it is a debtor because $b(g)>0$; and if $g=\bar{g}$ it is neither a debtor nor a creditor.

Two countervailing forces are at work: a high ratio of government expenditure, above the level consistent with the debt target $\bar{g}$, increases the level of debt, but on the other hand, as government expenditures are productive, they tend to increase tax returns on government bonds possibly above the speed of the adjustment set by the fiscal rule. The first factor is measured by the wedge $g-\bar{g}$ and the second factor is measured by the wedge $r(g)-r(\tilde{g})$, because $\tau r(g)-\phi=\tau(r(g)-r(\tilde{g}))$. Consequently, if $\bar{g}>\tilde{g}(\bar{g}<\tilde{g})$ then function $b(g)$ is increasing (decreasing) with $g$. It is also increasing if $\bar{g}=\tilde{g}$ and $\alpha>1 / 2$.

The next Lemma characterizes the debtor position of the government depending on the value of the two parameters defining the fiscal rule $\phi$ and $\bar{b}^{4}$ :

Lemma 1 (Government debtor position). Let equation (8) represent the government debtor position as a function of $g, b(g)$. Then the following cases are possible:

1. if $\phi \geq \max \{\alpha \tau,(1-\tau) / \bar{b}\}$ then $\min \{\bar{g}, \tilde{g}\} \geq 1$ then $b(g)>0$ for all $g \in(0,1)$.

2. if $(1-\tau) / \bar{b} \leq \phi<\alpha \tau$ then $\tilde{g}<1 \leq \bar{g}$ and $b(g)>0$ for $0<g<\tilde{g}$ and $b(g)<0$ for $\tilde{g}<g<1$

3. if $\alpha \tau<\phi \leq(1-\tau) / \bar{b}$ then $\bar{g} \leq 1<\tilde{g}$ which implies that $b(g)>0$ for $0<g<\bar{g}$ and $b(g)<0$ for $\bar{g}<g<1$;

4. if $\phi<\min \{\alpha \tau,(1-\tau) / \bar{b}\}$ then $\max \{\bar{g}, \tilde{g}\}<1$ then $b(g)<0$ if $\min \{\bar{g}, \tilde{g}\}<g<$ $\max \{\bar{g}, \tilde{g}\}$ and $b(g)>0$ otherwise.

Figure 1 illustrates Lemma 1, by showing the values of the policy parameters associated with the four cases.

In all four cases, if $g$ is close to zero the government will be a debtor. When $g$ is high the debtor position depends on the parameters: if both parameters are high the government should be a debtor (case L1(1)), but when they are both low it should be a creditor (case $L 1(4))$. In the other two cases, for a high debt target and a low speed of attainment, $L 1(2)$, or for a low debt target and a high speed, $L 1(3)$, there is an interval, $g \in(\min \{\bar{g}, \tilde{g}\}, \min \{\max \{\bar{g}, \tilde{g}\}, 1\})$, such that the government should be a creditor and a debtor outside that interval.

\footnotetext{
${ }^{4}$ All the proofs are in the Appendix.
} 


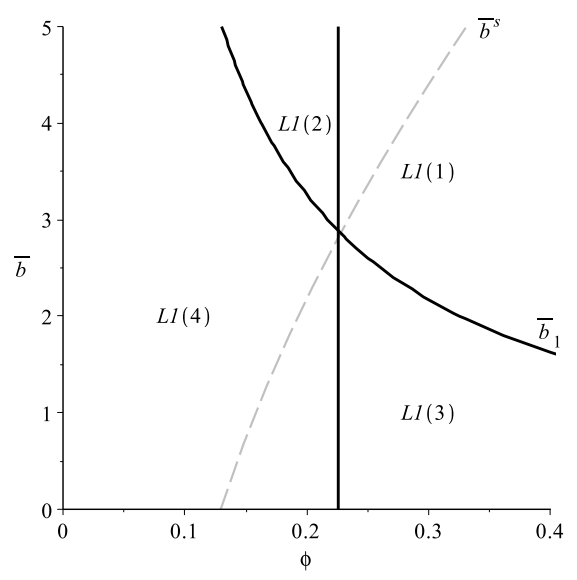

Figure 1: Graphical illustration of Lemma 1 in the space $(\phi, \bar{b})$ for $\alpha=0.65$ and $\tau=0.35$. We set $\bar{b}^{1} \equiv \frac{1-\tau}{\phi}$

\subsection{The DGE in detrended variables}

The rate of growth of the debt ratio can be obtained from two different sources. From the definition of $b$ we get

$$
\frac{\dot{b}}{b}=\frac{\dot{B}}{B}-\frac{\dot{Y}}{Y}=\gamma_{B}(g)-\gamma_{K}(g)-\left(\frac{1-\alpha}{\alpha}\right) \frac{\dot{g}}{g}
$$

where, from equation (3), the rate of growth of the government debt is

$$
\frac{\dot{B}}{B}=\gamma_{B}(g)=(1-\tau) r(g)+\frac{g-\tau}{b(g)} .
$$

However, we can also time-differentiate the feedback mechanism introduced by the fiscal rule in equation (8) to get

$$
\frac{\dot{b}}{b}=\frac{\left(\tau r(g)-\phi-(g-\bar{g}) \tau r^{\prime}(g)\right) g}{(\tau r(g)-\phi)(g-\bar{g})}\left(\frac{\dot{g}}{g}\right)
$$

Although we can eliminate either the rate of growth of the debt ratio or the expenditure ratio we only get an explicit differential equation if we eliminate the debt ratio, obtaining

$$
\left(\frac{\alpha g-\phi(1-\alpha) b(g)}{\alpha(g-\bar{g})}\right) \frac{\dot{g}}{g}=\frac{g-\tau}{b(g)}+(\alpha(1-\tau)+g-1) A(g)+z,
$$


where $z \equiv C / K$ denotes consumption detrended by the capital stock. From this equation we get the the rate of growth of the government expenditure ratio

$$
\frac{\dot{g}}{g} \equiv \gamma_{g}(g, z)=\frac{\psi(g, z)}{\delta(g)} .
$$

The numerator contains three wedges,

$$
\psi(g, z) \equiv \alpha(\tau r(g)-\phi)(g-\bar{g})(z-\zeta(g))
$$

the speed wedge, $\operatorname{\tau r}(g)-\phi$, the level wedge, $g-\bar{g}$, and a consumption wedge, $z-\zeta(g)$, where

$$
\zeta(g) \equiv(1-g-\alpha(1-\tau)) A(g)+(\tau-g) \frac{\tau r(g)-\phi}{g-\bar{g}},
$$

measures the potential for financing government expenditures out of consumption. The denominator is also a function of the level and speed wedges:

$$
\delta(g) \equiv \alpha g(\tau r(g)-\phi)-\phi(1-\alpha)(g-\bar{g})
$$

The rate of growth for private capital is a function of $g$ and $z$

$$
\frac{\dot{K}}{K}=\gamma_{K}(g, z) \equiv(1-g) A(g)-z
$$

Using equations (7) and (17) we find the rate of growth of the consumption-capital ratio

$$
\frac{\dot{z}}{z}=\gamma_{z}(g, z)=\gamma(g)-\gamma_{K}(g, z)=z-z(g),
$$

is equal to the wedge between consumption and potential consumption allowed for the level of the government expenditures,

$$
z(g) \equiv 1-g-\gamma(g)=\frac{(\sigma(1-g)-\alpha(1-\tau)) A(g)+\rho}{\sigma} .
$$

Function $z(g)$ is usually interpreted as a Laffer-curve associating government expenditures to the rate of growth of the economy. It has an inverted- $U$ shape, starting from $z(0)=\rho / \sigma>0$, reaching a maximum at a point where $g=(1-\alpha)(\sigma-\alpha(1-\tau)) / \sigma>0$ and decreasing for higher values of $g$ (possibly becoming negative for large $g$ ). There are two effects involved: first, productive government expenditures increase capital productivity thus having a positive effect on output and on the rate of capital accumulation, but second, they are financed by capital income taxation via the interest rate, which is also increasing in $g$. For low values of $g$ the first effect dominates and for high values of $g$ the second effect dominates. 
The DGE representation in detrended variables is the the path $(g(t), z(t))_{t \in[0, \infty)}$, such that every $(g(t), z(t)) \in \Omega$, where the domain of the detrended variables is $\Omega=(0,1) \times \mathbb{R}_{++}$, and are solutions of the system

$$
\begin{aligned}
& \dot{g}=g \frac{\psi(g, z)}{\delta(g)} \\
& \dot{z}=z(z-z(g))
\end{aligned}
$$

together with the initial conditions and the transversality condition. We will only consider DGE paths which are equilibrium BGP paths or converge asymptotically to an equilibrium BGP path.

An equilibrium BGP is a DGE path satisfying $\left(C^{*}(t), K^{*}(t), B^{*}(t)\right)_{t \in[0, \infty)}$ such that $C^{*}(t)=c^{*} e^{\gamma\left(g^{*}\right) t}, K^{*}(t)=k^{*} e^{\gamma_{K}\left(g^{*}, z^{*}\right) t}$ and $B^{*}(t)=b^{*} e^{\gamma_{B}\left(g^{*}, z^{*}\right) t}$ where $\left(g^{*}, z^{*}\right)$ are steady states of the system (20)-(21). A sufficient condition for the verification of the transversality condition is that asymptoticaly we should have $\rho+\sigma \gamma\left(g^{*}\right)-\gamma_{K}\left(g^{*}, z^{*}\right)>0$ and $\rho+\sigma \gamma\left(g^{*}\right)-\gamma_{B}\left(g^{*}, z^{*}\right)>0$.

The structure of this model is similar to the one found in Kamiguchi and Tamai (2012) and (Greiner and Fincke, 2015, p. 169-173). In particular both papers include a function similar to $\delta(g)$ that could take a zero value. If this is the case, function $\gamma_{g}(g, z)$ is not Lipschitzian. This implies that the existence and uniqueness of solutions for $t \in[0, \infty)$ of system (20)-(21) is not guaranteed, and therefore a DGE path may not exist.

We call impasse point to $\left(g^{s}, z^{s}\right) \in \Omega$ such that $\delta\left(g^{s}\right)=0^{5}$. A point $(g, z) \in \Omega$ such that $\delta(g) \neq 0$ will be called a regular point.

The set of impasse points, or impasse-set, is the one-dimensional manifold over $\Omega$,

$$
\mathcal{S}=\{(g, z) \in \Omega: \delta(g)=0\} .
$$

If $\mathcal{S}$ is non-empty (that is, if impasse points exist) they can be reached in finite or in infinite time. At any impasse point the rate of growth $\gamma_{g}$ becomes locally infinitely valued (and the jacobian of the system (20)-(21) has locally infinitely-valued eigenvalues). We call singular steady state to a steady state lying at the impasse surface. Observe that this case is non-generic, as it only occurs for specific parameter values. In the generic case in which the impasse point is not a steady state, it is reached at a particular finite time, following a sequence of regular points, but the solution may not be continued after it is reached. If there is no continuation any trajectory passing through such an impasse point cannot be a DGE path, and there is an existence problem which does not occur in regular DGE models. If there is continuation the determinacy properties of a crossing DGE path change in ways that also do not occur in regular models.

\footnotetext{
${ }^{5}$ As function $\delta(g)$ can only be equal to zero at a specific value for the state variables and not for a particular value of the parameters we have impasse-singularities and not fast-slow singularities (see Brito et al. (2017a)).
} 
It is shown in Brito et al. (2017a) that there are two local necessary conditions for the existence of singular DGE paths: crossing trajectories and at least one steady state should both exist. There are also global conditions that should be met. We address the local conditions in the next section and the global conditions in section 4 . In addition to singular DGE paths, regular DGE paths that do not cross the singular surface $\mathcal{S}$, can also exist. However, they are bounded to a subset of space $\Omega$ and cannot have points in the whole space as in DGE models without singularities.

\section{Local analysis}

The first subsection characterizes the impasse surface and the second the set of steady states of system (20)-(21).

\subsection{Impasse-surface}

If $\mathcal{S}$ is non-empty it partitions the domain of $(g, z), \Omega$, into three subsets $\mathcal{S}, \Omega_{+}$, containing points where $\delta(g)>0$, and $\Omega_{-}$, where $\delta(g)<0$.

In order to check for the existence and location of impasse points we need to define two critical values for $\bar{b}$

$$
\begin{aligned}
& \bar{b}^{s}=\frac{1}{\phi}(\tilde{g}-\tau)=\frac{1}{\phi}\left(\left(\frac{\phi}{\alpha \tau}\right)^{\frac{\alpha}{1-\alpha}}-\tau\right) \\
& \bar{b}_{1}^{s}=\frac{\phi(1-\tau+\alpha \tau)-\alpha^{2} \tau}{(1-\alpha) \phi^{2}}
\end{aligned}
$$

which are different except at point $(\phi, \bar{b})=(\alpha \tau,(1-\tau) /(\alpha \tau))$.

Lemma 2 (Singular points and partition of $\Omega$ ). Consider function $\delta(g)$ in equation (16). It is a convex function such that

1. if $\bar{b}>\bar{b}^{s}$ or if $\phi \geq \alpha \tau$ and $\bar{b}_{1}^{s}<\bar{b}<\bar{b}^{s}$ then the set $\mathcal{S}$ is empty and $\Omega=\Omega_{+}$;

2. if $0<\bar{b}<\bar{b}_{1}^{s}$ then there is one unique singular point $g^{s} \in(0, \min \{\tilde{g}, 1\})$ and the set $\Omega$ is partitioned into two regular subsets $\Omega_{+}=\left\{(g, z): 0<g<g^{s}\right\}$ and $\Omega_{-}=\{(g, z)$ : $\left.g^{s}<g<1\right\}$, and the impasse subset $\mathcal{S}=\left\{(g, z): g=g^{s}\right\}$;

3. if $\phi<\alpha \tau$ and $\bar{b}_{1}^{s}<\bar{b}<\bar{b}^{s}$ then there are two singular points $g_{1}^{s}$ and $g_{2}^{s}$, such that $0<g_{1}^{s}<\bar{g}<\tilde{g}<g_{2}^{s}<1$ and the set $\Omega$ is partitioned into three regular subsets $\Omega_{+, 1}=$ $\left\{(g, z): 0<g<g_{1}^{s}\right\}, \Omega_{+, 2}=\left\{(g, z): g_{2}^{s}<g<1\right\}$ and $\Omega_{-}=\left\{(g, z): g_{1}^{s}<g<g_{2}^{s}\right\}$, and two impasse subsets $\mathcal{S}_{1}=\left\{(g, z): g=g_{1}^{s}\right\}$ and $\mathcal{S}_{2}=\left\{(g, z): g=g_{2}^{s}\right\}$; 


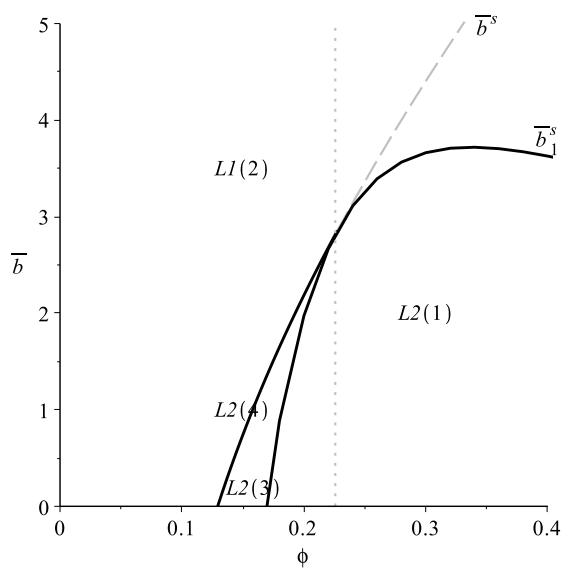

Figure 2: Graphical illustration of Lemma 2 in the space $(\phi, \bar{b})$ for $\alpha=0.65$ and $\tau=0.35$.

4. if $\phi<\alpha \tau$ and $\bar{b}=\bar{b}^{s}$ then there is one unique singular point such that $g^{s}=\bar{g}=\tilde{g}<1$ and the set $\Omega$ is partitioned into the regular set $\Omega_{+}=\left\{(g, z): g \neq g^{s}\right\}$ and the impasse set $\mathcal{S}=\left\{(g, z): g=g^{s}\right\}$. This is a non-generic case.

Figure 2 illustrates Lemma 2, by showing the values of the policy parameters associated with the four cases, labelled $L 2(1), L 2(2), L 2(3)$ and $L 2(4)$ (which corresponds to curve $\bar{b}=\bar{b}^{s}$ for $\left.\phi<\alpha \tau\right)$.

A necessary condition for the existence of singular points is $\bar{g} \leq \tilde{g}$ which is equivalent to $\bar{b} \leq \bar{b}^{s}$. In Figure 2 this corresponds to the points below curve $\bar{b}=\bar{b}^{s}$. This means that in that Figure the points above curve curve $\bar{b}=\bar{b}^{s}$ represent the combinations of fiscal rule parameters yielding $\bar{g}>\tilde{g}$.

From Lemma 2 we conclude that, if the debt target is set at relatively low (high) levels or the speed of convergence is high (low), there will (not) exist impasse points and the associated singular dynamics will be absent. Impasse points exist only in the generic cases $L 2(1)$ and $L 2(2)$ and in the non-generic case $L 2(4)$. Comparing with Lemma 1, observe that the government can be a creditor only if there is an impasse surface and the variables $(g, z)$ lie within set $\Omega_{-}$. If the impasse set is empty (as in the $L 2(1)$ case) the government is always a debtor.

From Lemmas 1 and 2 we can also extract some intuition for the speed of the adjustment of the government debt position for different values of $g$. The elasticity of the creditor position is

$$
\beta(g) \equiv-\frac{b^{\prime}(g) g}{b(g)}=\frac{1-\alpha}{\alpha}-\frac{\delta(g)}{(\tau r(g)-\phi)(g-\bar{g})},
$$

where the second term has the same sign as function $b(g)$. We can easily infer the following: first, at an impasse point the elasticity $\beta($.$) is equal to the share of public expenditures$ 
versus private capital in output, $(1-\alpha) / \alpha$; second inside subsets $\Omega_{+}$, where the government is always a debtor, the elasticity is lower than $(1-\alpha) / \alpha$; third inside subset $\Omega_{-}$the elasticity is higher (lower) if the government is a debtor (creditor). This means that trajectories that cross $\mathcal{S}$, if they exist, witness a large change in the reaction of the creditor position after a relatively small change in $g$. This means that the rule can introduce non-monotonous reactions in the government budget.

We now characterize the local dynamics for points within $\mathcal{S}$ when it is non-empty (that is when $\bar{g} \leq \tilde{g})$. There are four types of impasse points. First, if $\delta^{\prime}\left(g^{s}\right) \psi\left(g^{s}, z^{s}\right)<0$ point $\left(g^{s}, z^{s}\right) \in \mathcal{S}$ is an attractor point and we call it black-hole because this points attracts neighbouring trajectories which reach $\mathcal{S}$ in finite time and does not allow them to be continued afterwards. This means that trajectories hitting a black-hole cannot be DGE trajectories. Second, if $\delta^{\prime}\left(g^{s}\right) \psi\left(g^{s}, z^{s}\right)>0$ point $\left(g^{s}, z^{s}\right) \in \mathcal{S}$ is an attractor point and we call it white-hole because this points repels neighbouring trajectories allowing them to be continued in time. Although they can be DGE trajectories they cannot cross $\mathcal{S}$, which means that they remain confined to subset $\Omega_{-}$or to subset $\Omega_{+}$. Those points would not belong to DGE paths if crossing $\mathcal{S}$ is necessary to reach a steady state. Third, if $\delta^{\prime}\left(g^{s}\right) \psi\left(g^{s}, z^{s}\right)=0$, under the conditions $\psi\left(g^{s}, z^{s}\right)=0$ and $\delta^{\prime}\left(g^{s}\right) \neq 0$, we have an impasse-transversal point. An impasse transversal point can be seen as a fixed point of the de-singularized dynamic system

$$
\begin{aligned}
& \dot{g}=g \psi(g, z) \\
& \dot{z}=z \delta(g)(z-z(g))
\end{aligned}
$$

where the vector field has the same integral curves as system (20)-(21), but we introduce a time-reversal for the integral curves within set $\Omega_{-}$, and remove the singularity ${ }^{6}$. From now on we denote the impasse-transversal points by $\left(g^{i}, z^{i}\right)$ and denote the set of impassetransversal points by

$$
\Gamma_{I}=\{(g, z) \in \mathcal{S}: z=\zeta(g)\}
$$

where $\zeta(g)$ is defined in equation (15). Every impasse-transversal point, $\left(g^{i}, z^{i}\right)=\left(g^{s}, \zeta\left(g^{s}\right)\right)$, can be classified further depending on their local dynamic properties, which can be determined from the eigenvalues of the Jacobian of the de-singularized system evaluated at an impasse-transversal point

$$
J^{i}=J\left(g^{i}, z^{i}\right)=\left(\begin{array}{cc}
g^{i} \psi_{g}\left(g^{i}, z^{i}\right) & g^{i} \psi_{z}\left(g^{i}, z^{i}\right) \\
z^{i} \delta^{\prime}\left(g^{i}\right)\left(z^{i}-z\left(g^{i}\right)\right) & 0
\end{array}\right)
$$

There are three types of impasse-transversal points: First, point $\left(g^{i}, z^{i}\right)$ is a transversal saddle if $J^{i}$ has two real eigenvalues, one negative and one positive. Through this point

\footnotetext{
${ }^{6}$ Using the analogy with fast-slow singularities, we can see desingularization as a device to uncover the singularities by locally changing the time scale such that the speed of the trajectories close to the singular surface becomes finite.
} 
there are two trajectories crossing $S$, one coming from $\Omega_{+}$with a continuation in $\Omega_{-}$, and another coming from $\Omega_{-}$with a continuation in $\Omega_{+}$. Second, point $\left(g^{i}, z^{i}\right)$ is a transversal node if $J^{i}$ has two real eigenvalues with the same sign. Transversal nodes have a basin of attraction belonging to set $\Omega_{+}$, if the eigenvalues are negative, or to set $\Omega_{-}$, if the eigenvalues are positive. There are infinitely many trajectories coming from that basin of attraction and having a continuation in the opposite subset. Third, point $\left(g^{i}, z^{i}\right)$ is a transversal focus if $J^{i}$ has two complex-conjugate eigenvalues. In this case there are no crossing trajectories. We say we have worm-hole dynamics, meaning that there are crossing trajectories, in the first two cases and these are the only cases in which there could exist crossing DGE paths ${ }^{7}$.

In Lemma 2 we proved that one or two impasse points can exist, i.e $\mathcal{S}$ is non-empty. Impasse-transversal points further introduce a partition over set $\mathcal{S}$ into a subset of attractor points, $\mathcal{S}^{-}$, and a subset of repeller points, $\mathcal{S}^{+}$, which determine the existence and the type of DGE paths in their neighborhood. In Lemma 3 we determine the existence and number of impasse-transversal points, and the partitions they introduce over $\mathcal{S}$ and in Lemma 4 we characterize the types of impasse-transversal points (i.e, we partition further $\Gamma_{I}$ ).

Lemma 3 (Partition of the impasse set). Let

$$
\bar{b}^{i}=\{\bar{b}: \delta(g, \bar{b})=0, \zeta(g, \bar{b})=0\} .
$$

and assume $\bar{b}<\bar{b}_{s}$. Five cases are possible:

1. If $\phi>\alpha \tau$ and $\bar{b}^{i} \leq \bar{b}<\bar{b}_{1}^{s}$ then set $\mathcal{S}$ has only one component, $\mathcal{S}_{1}$, and contains only attractor points. Then $\mathcal{S}=\mathcal{S}_{1}^{-}$;

2. If $0<\bar{b}<\min \left\{\bar{b}^{i}, \bar{b}_{1}^{s}\right\}$ then set $\mathcal{S}$ has only one component, $\mathcal{S}_{1}$, containing one impassetransversal point, $\left(g^{i}, z^{i}\right)=\left(g_{1}^{s}, \zeta\left(g_{1}^{s}\right)\right)$, dividing $\mathcal{S}_{1}$, into a subset of attractor points, if $z>z^{i}$, and a subset of repeller points, if $z<z^{i}$. Then $\mathcal{S}=\mathcal{S}_{1}^{-} \cup \Gamma_{I} \cup \mathcal{S}_{1}^{+}$;

3. If $\phi<\alpha \tau$ and $\max \left\{\bar{b}_{1}^{s}, \bar{b}^{i}\right\} \leq \bar{b} \leq \bar{b}^{\text {s }}$ then set $\mathcal{S}$ has two components, $\mathcal{S}_{1}$, containing only attractor points, and $\mathcal{S}_{2}$, containing only repeller points. Then $\mathcal{S}=\mathcal{S}_{1}^{-} \cup \mathcal{S}_{2}^{+}$;

4. If $\phi<\alpha \tau$ and $\max \left\{0, \bar{b}_{1}^{s}\right\} \leq \bar{b} \leq \bar{b}^{i}$ then set $\mathcal{S}$ has two components, $\mathcal{S}_{1}$ and $\mathcal{S}_{2}$. Set $\mathcal{S}_{1}$, contains one impasse-transversal point, $\left(g^{i}, z^{i}\right)=\left(g_{1}^{s}, \zeta\left(g_{1}^{s}\right)\right)$ dividing $\mathcal{S}_{1}$ into a subset of repeller points, if $z<z^{i}$, and attractor points, if $z>z^{i}$, and set $\mathcal{S}_{2}$ only contains repeller points. Then $\mathcal{S}=\mathcal{S}_{1}^{-} \cup \Gamma_{I, 1} \cup \mathcal{S}_{1}^{+} \cup \mathcal{S}_{2}^{+}$;

5. If $\phi<\alpha \tau$ and $\max \left\{0, \bar{b}^{i}\right\}<\bar{b}<\bar{b}^{s}$ then set $\mathcal{S}$ has two components, $\mathcal{S}_{1}$ and $\mathcal{S}_{2}$ and every subset contains one impasse-transversal point. Set $\mathcal{S}_{1}$, contains one impasse-transversal

\footnotetext{
${ }^{7}$ See Brito et al. (2017a) for further details. There is another type of impasse point, impasse-tangent points at which a tangent trajectory passes through $\mathcal{S}$. In the present model there are no impasse-tangent points.
} 


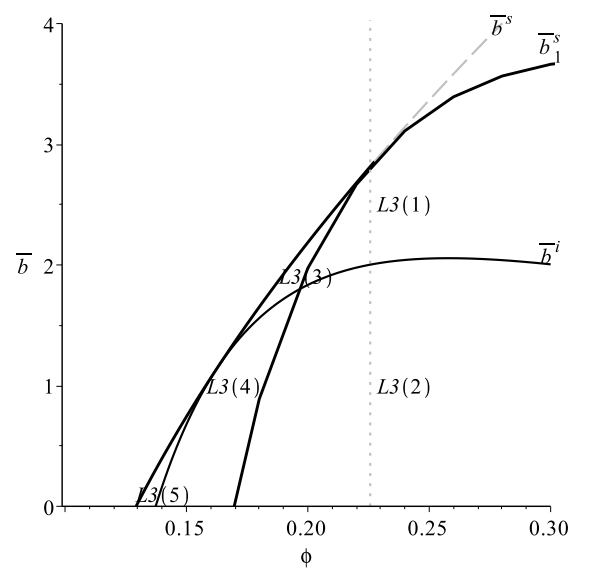

Figure 3: Graphical illustration of Lemma 3 in the space $(\phi, \bar{b})$ for $\alpha=0.65$ and $\tau=0.35$.

point, $\left(g_{1}^{i}, z_{1}^{i}\right)=\left(g_{1}^{s}, \zeta\left(g_{1}^{s}\right)\right)$ dividing $\mathcal{S}_{1}$ into a subset of repeller points, if $z<z_{1}^{i}$, and a set of attractor points, if $z>z_{1}^{i}$, and set $\mathcal{S}_{2}$ also contains one impasse-transversal point, $\left(g_{2}^{i}, z_{2}^{i}\right)=\left(g_{2}^{s}, \zeta\left(g_{2}^{s}\right)\right)$, dividing it into a subset of repeller points, if $z>z_{2}^{i}$, and attractor points, if $z<z_{2}^{i}$. Then $\mathcal{S}=\mathcal{S}_{1}^{-} \cup \Gamma_{I, 1} \cup \mathcal{S}_{1}^{+} \cup \mathcal{S}_{2}^{-} \cup \Gamma_{I, 2} \cup \mathcal{S}_{2}^{+}$.

Figure 3 illustrates Lemma 3, by depicting the values of the policy parameters associated with the five cases. There are no impasse-transversal points in cases $L 3(1)$, in which there is a single impasse-surface, and L3(3), in which there are two impasse-surfaces. There is one impasse transversal point in cases $L 3(2)$ and $L 3(4)$, and there are two impasse-transversal points in case $L 3(5)$. The last case occurs for relatively low values of the two rule parameters: low target value for the debt ratio and low speed of adjustment.

When there is a single impasse-transversal point it will belong to set $\mathcal{S}_{1}$ which is associated to relatively low levels of $g$ and to a debtor position. When there are two impasse-transversal points they belong to two different branches of set $\mathcal{S}$, one for low levels of $g$ and another for high values of $g$ and both correspond to debtor positions.

This model produces different configurations as compared to Brito et al. (2017a), in which there is a single impasse surface and at most one impasse-transversal point, and to Brito et al. (2017b), in which there can be two impasse-transversal points but within a single impasse surface.

Lemma 4 (Characterization of impasse-transversal points). Let

$$
\bar{b}_{d}^{i}=\left\{\bar{b}: \delta\left(g_{d}^{i}(\bar{b}), \bar{b}\right)=0\right\}
$$

where

$$
g_{d}^{i} \equiv \frac{\phi(1-\alpha)\left((1-\tau)(\sigma-1) \bar{g}+\sigma \tau^{2}\right)}{\rho+\alpha \tau+\phi(\sigma(1-\alpha \tau)-(1-\tau))},
$$


and let

$$
\bar{b}_{\Delta}^{i}=\left\{\bar{b}: \Delta J^{i}(\bar{b})=0\right\} .
$$

where $\Delta J^{i}(\bar{b})=\Delta J\left(g^{i}(\bar{b}), \zeta\left(g^{i}(\bar{b})\right)\right)$ is the discriminant of the Jacobian of the desingularized system evaluated at an impasse-transversal point. For reasonable parameter values there are two points $\bar{b}_{\Delta, 1}^{i}<\bar{b}_{\Delta, 2}^{i}$. Assume that $\sigma \geq 1$ and $\bar{b}<\bar{b}^{s}<\tau(\sigma \phi-\rho) /(\rho \phi)$. Then eight cases are possible:

1. if $\bar{b}_{b}^{i}<\bar{b}<\bar{b}^{i}$ then there is one impasse-transversal saddle-point $\left(g^{i}, z^{i}\right)$ such that $g^{i}<\bar{g}$;

2. if $\bar{b}_{\Delta}^{i}<\bar{b}<\bar{b}_{d}^{i}$ then there is one impasse-transversal node $\left(g^{i}, z^{i}\right)$ such that $g^{i}<\bar{g}$;

3. if $0<\bar{b}<\bar{b}_{\Delta, 1}^{i}$ then there is one impasse-transversal focus $\left(g^{i}, z^{i}\right)$ such that $g^{i}<\bar{g}$;

4. if $\bar{b}_{b}^{i}<\bar{b}^{i}<\bar{b}<\bar{b}_{\Delta, 2}^{i}$ then there are two impasse-transversal points, $\left(g_{1}^{i}, z_{1}^{i}\right)$ and $\left(g_{2}^{i}, z_{2}^{i}\right)$, such that $g_{1}^{i}<\bar{g}<\tilde{g}<g_{2}^{i}$, where the first is a saddle and the second a node;

5. if $\bar{b}_{\Delta, 2}^{i}<\bar{b}<\bar{b}^{s}$ then there are two impasse-transversal points, $\left(g_{1}^{i}, z_{1}^{i}\right)$ and $\left(g_{2}^{i}, z_{2}^{i}\right)$, such that $g_{1}^{i}<\bar{g}<\tilde{g}<g_{2}^{i}$, where the first is a saddle and the second a focus;

6. if $\bar{b}^{i}<\bar{b}_{\Delta, 1}^{i}<\bar{b}<\min \left\{\bar{b}_{d}^{i}, \bar{b}_{\Delta, 2}^{i}\right\}$ then there are two impasse-transversal points, $\left(g_{1}^{i}, z_{1}^{i}\right)$ and $\left(g_{2}^{i}, z_{2}^{i}\right)$, such that $g_{1}^{i}<\bar{g}<\tilde{g}<g_{2}^{i}$, where the two are transversal nodes;

7. if $\bar{b}^{i}<\bar{b}_{\Delta, 1}^{i}<\bar{b}_{\Delta, 2}^{i}<\bar{b}<\bar{b}_{d}^{i}$ then there are two impasse-transversal points, $\left(g_{1}^{i}, z_{1}^{i}\right)$ and $\left(g_{2}^{i}, z_{2}^{i}\right)$, such that $g_{1}^{i}<\bar{g}<\tilde{g}<g_{2}^{i}$, where the first is a node and the second is a focus;

8. if $\bar{b}^{i}<\bar{b}<\bar{b}_{\Delta, 1}^{i}<\bar{b}_{\Delta, 2}^{i}<\bar{b}_{d}^{i}$ there are two impasse-transversal points, $\left(g_{1}^{i}, z_{1}^{i}\right)$ and $\left(g_{2}^{i}, z_{2}^{i}\right)$, such that $g_{1}^{i}<\bar{g}<\tilde{g}<g_{2}^{i}$, where the two are transversal foci.

Figure 4 illustrates Lemma 4, by depicting the values of the policy parameters associated with the five cases $L 4(1)$ to $L 4(5)$. Cases $L 4(6), L 4(7)$ and $L 4(8)$ only occur if curves $\bar{b}=\bar{b}_{d}^{i}$ and $\bar{b}=\bar{b}_{\Delta}^{i}$ cross curve $\bar{b}=\bar{b}^{i}$, which only happen for $\sigma \approx 1$. This also means that there are values for the parameters such that a singularity induced bifurcation point can exist, such that $\bar{b}=\bar{b}^{s}=\bar{b}_{d}^{i}$, implying that $g_{s}^{*}=\bar{g}=\tilde{g}$, where $g_{s}^{*}$ is an impasse-transversal steady state.

While the existence and the partition of the impasse surface is independent of the elasticity of intertemporal substitution and the rate of time preference, these two parameters determine the type of impasse-transversal points and their local dynamic properties. That is, they determine the existence of crossing trajectories.

Referring to the cases in Lemma 4 and Figure 4 we can have the following types of crossing trajectories:

Proposition 1 (Crossing trajectories). Crossing trajectories exist in the following cases:

1. in case L4(1) there are two trajectories crossing the unique impasse surface one from $\Omega_{+}$towards $\Omega_{-}$and another in the opposite direction; 

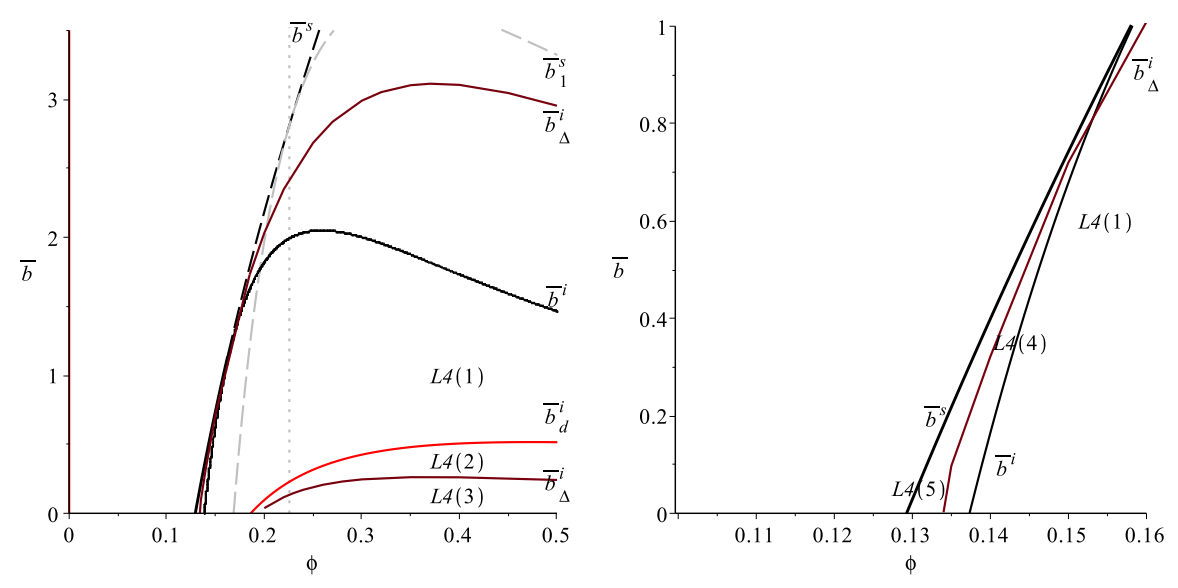

Figure 4: Graphical illustration of Lemma 4 in the space $(\phi, \bar{b})$ for $\alpha=0.65, \tau=0.35, \sigma=2$ and $\rho=0.02$. The RHS panel zooms a small area in the LHS panel to the left of area $L 4(1)$.

2. in case L4(2) and in case L4(7) there are, in most cases, an infinite number of trajectories crossing from $\Omega_{-}$to $\Omega_{+}$;

3. in case L4(4) there are two crossing trajectories, in both directions over set $\mathcal{S}_{1}$, between $\Omega_{-}$and $\Omega_{+, 1}$, and there are an infinite number of trajectories crossing $\mathcal{S}_{2}$ from $\Omega_{-}$to $\Omega_{+, 2}$;

4. in case $L 4(5)$ there are trajectories in infinite number going from $\Omega_{-}$to $\Omega_{+, 1}$ and from $\Omega_{-}$to $\Omega_{+, 2}$.

\subsection{Balanced growth paths and local dynamics}

An equilibrium regular BGP is a BGP such that the steady state, $\left(g^{*}, z^{*}\right)$, of system (20)-(21) is a point belonging to the set

$$
\Gamma_{E}=\left\{(g, z) \in(0,1) \times(0, \infty): \psi(g, z)=0, \gamma_{z}(g, z)=0, \delta(g) \neq 0\right\}
$$

and verifying the transversality condition. An equilibrium singular BGP is a BGP such that the steady state $\left(g_{s}^{*}, z_{s}^{*}\right)$ is a point belonging to the set

$$
P_{E}=\left\{(g, z, \bar{b}) \in(0,1) \times(0, \infty)^{2}: \psi(g, z)=0, \gamma_{z}(g, z)=0, \delta(g)=0\right\}
$$

and verifying the transversality condition ${ }^{8}$.

\footnotetext{
${ }^{8}$ Observe that given the constraint introduced by the singularity condition, the set of singular steady states has a higher co-dimension in the joint space of the variables and the parameters.
} 
We introduce three new critical values for the debt ratio target parameter, $\bar{b}$ :

$$
\begin{gathered}
\bar{b}_{1}^{*} \equiv \frac{\tau(\sigma \phi-\rho)}{\rho \phi}, \\
\bar{b}_{z}^{*} \equiv\{\bar{b}: z(g, \bar{b})=0, \tau r(g)=\varphi(g, \bar{b})\}
\end{gathered}
$$

and

$$
\bar{b}_{s}^{*} \equiv\left\{\bar{b}: \delta\left(g_{s}^{*}(\bar{b}), \bar{b}\right)=0\right\},
$$

where $g_{s}^{*}=g_{d}^{i}$ (see equation (27)).

The next result characterises the equilibrium BGP's as regards their number, their location in the partition introduced by the impasse surface(s) and the asset position of the government.

Lemma 5 (Number and location of BGPs). Let $\bar{b}_{s} \bar{b}_{1}^{*}, \bar{b}_{z}$ and $\bar{b}_{s}^{*}$ be given by equations (23), (28), (29) and (30), respectively, and assume that $\rho<\alpha(1-\tau)$. Then the following cases are possible, concerning the number and the location of steady states $\left(g^{*}, z^{*}\right)$ :

1. if $\bar{b}<\bar{b}_{1}^{*}$ then there is one isolated regular BGP such that $g^{*}<g_{1}^{s}$ is in $\Omega_{+}$and the government is a creditor;

2. if $\bar{b}>\max \left\{\bar{b}_{z}, \bar{b}_{s}^{*}\right\}$ then there is one isolated regular BGP such that $g_{1}^{s}<g^{*}<g_{1}^{s}$ is in $\Omega_{+}$and the government is a debtor;

3. if $\bar{b}_{z}<\bar{b}<\bar{b}_{s}^{*}$ then there is one isolated regular BGP such that $\bar{g}<g^{*}<\tilde{g}$ is in set $\Omega_{-}$ and the government is a debtor;

4. if $\bar{b}=\bar{b}_{s}^{*} \geq \bar{b}_{z}$ then there is one isolated singular BGP such that $g^{*}=g_{s}^{*}$ is in $\mathcal{S}$ which corresponds to a debtor position;

5. if $\bar{b}^{s}<\bar{b}<\max \left\{\bar{b}_{1}^{*}, \bar{b}_{z}^{*}\right\}$ then there are two regular BGP's such that $g_{1}^{*}<g_{2}^{*}<g_{1}^{s}$ are both in $\Omega_{+}$and $g_{1}^{*}$ corresponds to a debtor and $g_{2}^{*}$ to a creditor position;

6. if $\bar{b}_{s}^{*}<\bar{b}<\max \left\{\bar{b}^{s}, \bar{b}_{z}^{*}\right\}$ then there are two regular BGP's such that $g_{1}^{*}<g_{1}^{s}<g_{2}^{*}, g_{1}^{*}$ is in $\Omega_{+}$and corresponds to a debtor position, while $g_{2}^{*}$ is in $\Omega_{-}$and corresponds to a creditor position;

7. if $0<\bar{b}<\min \left\{\bar{b}_{s}^{*}, \bar{b}_{z}^{*}\right\}$ then there are two regular BGP's such that $g_{1}^{s}<g_{1}^{*}<g_{2}^{*}$, where both are in $\Omega_{-}$and $g_{1}^{*}$ corresponds to a debtor position and $g_{2}^{*}$ to a creditor position;

8. if $\bar{b}=\bar{b}_{s}^{*}<\bar{b}_{z}^{*}$ then there is one singular $B G P$, such that $g_{1}^{*}=g_{s}^{*}$ is in $\mathcal{S}$ and a regular $B G P$ such that $g_{s}^{*}<g_{2}^{*}$ is in $\Omega_{-}$and corresponds to a debtor position. 


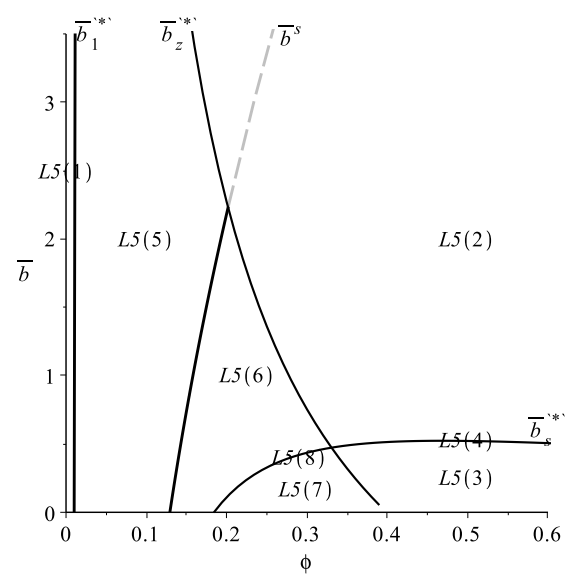

Figure 5: Graphical illustration of Lemma 5 in the space $(\phi, \bar{b})$ for $\alpha=0.65$ and $\tau=0.35$, $\sigma=2$ and $\rho=0.02$

Figure 5 presents a graphical illustration of Lemma 5 for different values of $\bar{b}$ and $\phi$, and given values of the other parameters, including $\sigma$ and $\rho$. A unique BGP exists for cases $L 5(1)$ to $L 5(4)$ and two BGP's can exist in the other cases. In cases $L 5(4)$ and $L 5(8)$ there is a singular BGP, isolated in the first case and jointly with a regular BGP in the second case. Multiple equilibrium BGP's tend to occur for relatively low (high) values of the speed of the adjustment and high (low) levels for the debt target. Singular BGP's are located in the unique impasse surface $\mathcal{S}$ when they are isolated or are in surface $\mathcal{S}_{1}$ when they are not. They exist for relatively high levels for the speed of adjustment and for a wide interval of values for the debt target. Given benchmark parameter values for the tax rate, the intertemporal elasticity of substitution and the rate of time preference, a singular BGP can occur for a target debt ratio set around or below 50\%, thus very close to the Fiscal Compact target.

When there are multiple steady states, the BGP associated to the higher value for $g, g_{2}^{*}$, displays a higher rate of growth, because the rate of growth is an increasing function of $g$ (see equation (7)). However, as can be seen in the next section, this case corresponds to a very high expenditure ratio involving an almost confiscatory economy, which makes this BGP possibly dominated in utility terms by the adjacent BGP which has a lower expenditure ratio and a lower growth rate.

It is also possible to prove that $\sigma<1-\tau$ and $\bar{b}<0$ are necessary conditions for getting a steady state level of debt $b^{*}=b\left(g^{*}\right)=\bar{b}$. Therefore, for the accepted values of the parameters, although the target level influences the dynamics it does not correspond to a BGP outcome.

Next we study the local dynamics at equilibrium BGP's.

Lemma 6 (Local dynamics at a regular BGP). If $\bar{b}<\bar{b}_{s}^{*}$ then the regular steady state $\left(g_{1}^{*}, z_{1}^{*}\right)$, isolated or not, is a saddle-point. If $\bar{b}>\bar{b}_{s}^{*}$ then the steady state $\left(g_{1}^{*}, z_{1}^{*}\right)$, isolated or not, is an unstable node or focus. If it exists, the steady state $\left(g_{2}^{*}, z_{2}^{*}\right)$, is always an unstable node 
or focus.

Therefore, we have the following cases concerning asymptotic local dynamics, and therefore the asymptotic determinacy properties, of equilibrium BGP's:

Proposition 2 (Determinacy of BGP's). Consider the cases in Lemma 5. The following types of BGP dynamics are possible:

1. in case L5(3) the steady state $\left(g^{*}, z^{*}\right)$ is a saddle point, then the BGP is locally determinate;

2. in case $L 5(7)$ there are two steady states where $\left(g_{1}^{*}, z_{1}^{*}\right)$ is a saddle point and $\left(g_{2}^{*}, z_{2}^{*}\right)$ is locally unstable, then the first BGP is locally determinate and the second is overdeterminate;

3. in cases L5(1) and L5(2) the unique steady state is unstable, then the BGP is overdeterminate;

4. in cases $L 5(5)$ and $L 5(6)$ the two steady states are unstable, then the associated BGP's are over-determinate.

We say a steady state is over-determined if the dimension of the local stable manifold in the neighborhood of a steady state is less than one. This implies that the a DGE path only exists if $g(0)=g^{*}$. Then the initial values of the pre-determinate variables cannot be chosen freely and should belong to a one-dimensional manifold. Therefore, over-determinate steady states can only be DGE paths if the initial point and the steady state for the detrended variables coincide. If this is the case then over-determinacy means that the DGE and the BGP paths coincide and there is no transitional dynamics.

Two BGPs with the same local stability properties cannot exist in regular DGE models in which there are no impasse-singularities (see case 4 in Proposition 2). This property also occurs in (Greiner and Fincke, 2015, p. 169-173) and in Brito et al. (2017a). However, while in those papers there are two saddle-point steady states, in our case there are two unstable steady states.

Furthermore, cases $L 5(4)$ and $L 5(8)$ cannot exist in regular DGE models. If there is a singular steady state, and we consider the local dynamics in terms of the dynamic system (20)-(21), there will be a DGE path converging with infinite speed to a singular point. However we know that in this case the BGP path grows at a finite rate of growth $\gamma\left(g_{s}^{*}\right)$. In order to uncover the local dynamics we need to note that the value of the parameter leading to this case, $\bar{b}_{s}^{*}$, is equal to the value of the parameter associated to a zero determinant of the de-singularized vector field (25)-(26), $\bar{b}_{s}^{*}=\bar{b}_{d}^{i}$ (see Lemma 4). This means that it is a saddle-node of the desingularized vector field, implying there is convergence from the $\Omega_{-}$-side of the impasse-surface and divergence from the the $\Omega_{+}$-side. That is, the DGE path converges locally at a very high speed, and in an asymmmetric way, to a BGP path growing 
at rate $\gamma\left(g_{s}^{*}\right)$. This case corresponds to a bifurcation point introduced by the existence of an impasse singularity.

\section{Equilibrium dynamics}

Propositions 1 and 2 suggest that several types of DGE paths exist in our model: overdeterminate DGE which are coincident with one or two BGP's, determinate DGE paths converging to a BGP without crossing an impasse surface, or determinate DGE paths converging to a BGP after crossing an impasse surface or to a BGP lying in the impasse surface (we call these singular DGE paths).

A determinate DGE path involving crossing trajectories can only exist if specific both local and global specific conditions are jointly satisfied. There are two local conditions: at least one steady state in which $\left(g^{*}, z^{*}\right)$ is a saddle-point or a stable node or stable focus should exist in $\Omega_{+}$or $\Omega_{-}$, and the impasse surface, $\mathcal{S}$, at the boundary of those two subsets, contains at least one impasse-transversal saddle-point or node. There are also two global conditions: the initial point $(g(0), z(0))$ and the steady state belong to different subsets, $\Omega_{+}$or $\Omega_{-}$, and there is at least one (heteroclinic) trajectory joining the impasse-transversal point and the steady state, or joining the initial point and a singular steady-state.

The other type of DGE paths, over-determinate or determinate with no crossing, can be seen as cases in which either one of the local conditions (in the case of over-determinacy) or one of the global conditions (in the case of non-crossing DGE paths) fail.

At first look, it seems that the difference introduced by the existence of impasse-singularities, as regards regular models, is only related to the existence of the crossing DGE paths. However, this is not the case. The existence of impasse-singularities can also be noted in the existence of multiple over-determinate DGE paths and on the confinement of the existence of non-crossing DGE to a particular subset of the state space $\Omega$.

Next we gather some representative DGE dynamics that illustrate the effect of the existence of impasse-singularities introduced by the fiscal policy rule. In those examples we constrain the analysis to cases in which an impasse surface exists, that is $\bar{b} \leq \bar{b}^{s}$. From Propositions 1 and 2 we know that, for specific subsets of the parameters values, at least one of the local conditions holds, although we were unable to find parameter values such that determinate DGE paths with crossing trajectories exist. However, the existence of singularities still constrains the dynamics in significant ways.

\subsection{Multiple over-determined BGP's}

Most of the equilibrium DGE dynamics involve over-determinacy, that is the existence of BGP's isolated or multiple, in which the detrended variables are unstable nodes or foci, the equilibrium dynamics is reduced to the BGP and there is no transitional dynamics. These cases are possible only if the initial values for the pre-determined variables, $K$ and $B$, are in 


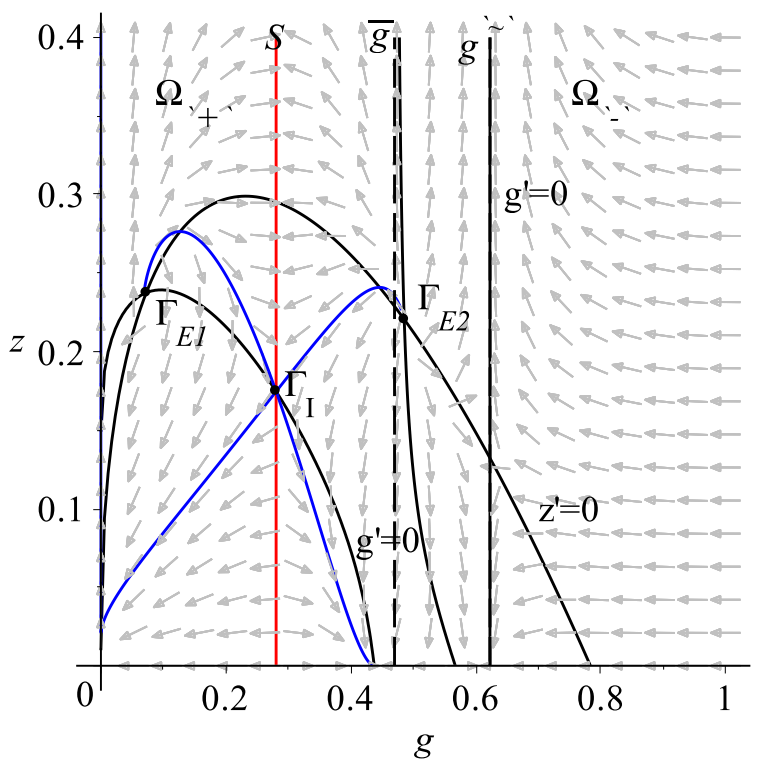

Figure 6: Phase diagram for the case L4(1) in Figure 4 and L5(6) in Figure 5, and with $\alpha=0.7, \sigma=2, \rho=0.02, \tau=0.35, \phi=0.2$ and $\bar{b}=0.6$.

a given proportion such that the initial values of the detrended variables belong to the set $\Gamma_{E}$. If there is a single steady state $\Gamma_{E}=\left\{\left(g^{*}, z^{*}\right)\right\}$, the initial values should verify $g(0)=g^{*}$ and $z(0)=z^{*}$. If there is multiplicity of steady states, $\Gamma_{E}=\left\{\left(g_{1}^{*}, z_{1}^{*}\right),\left(g_{2}^{*}, z_{2}^{*}\right)\right\}$ then there are two initial points with associated different equilibrium BGP's.

Figure 6 presents an example associated to reasonable parameter values corresponding to case $L 4(1)$ in Figure 4 and $L 5(6)$ in Figure 5. There are two steady states, labelled $\Gamma_{E_{1}}$ and $\Gamma_{E_{2}}$, both unstable, where the first is in set $\Omega_{+}$and corresponds to a debtor position and the second is in set $\Omega_{-}$and corresponds to a creditor position. In addition, observe that there is one impasse-transversal saddle point, $\Gamma_{I}$, and there are two crossing trajectories starting at each steady state and passing through the impasse-transversal point. None of those trajectories are equilibria, because they do not converge to a steady state. There is another crossing between the isoclines at point $\tilde{g}$ but it does not satisfy the transversality condition.

Again, this type of phase diagram cannot exist in regular DGE models. If there are two steady states, in regular models, they have different local dynamic properties, because the curvatures of the isoclines at the two steady states should be different. The existence of an impasse surface allows for the different curvatures of the isoclines at the two steady states to be associated to the same type of local dynamics. 


\subsection{Determinate BGP's}

There are some combinations of parameters that allow the local conditions for the existence of a determinate DGE, involving local dynamics convergent to one BGP, for parameter values such that $\bar{b} \leq \bar{b}^{s}=\bar{b}_{d}^{i}$ : cases $L 4(2), L 4(6)$ and $L 4(7)$ from Lemma 4 and $L 5(3), L 5(4), L 5(7)$ and $L 5(8)$ from Lemma 5.

However, for the cases represented in Figures 4 and 5 crossing trajectories cannot exist, because a trajectory joining the impasse-transversal point $\left(g_{1}^{i}, z_{1}^{i}\right)$ to $\left(g_{1}^{*}, z_{1}^{*}\right)$ is not possible. In order to prove this, observe that the first point is an impasse-transversal node point where crossing trajectories flow from $\Omega_{-}$to $\Omega_{+}$and the second is a saddle point belonging to $\Omega_{-}$. Indeed, the stable manifold associated to $\left(g_{1}^{*}, z_{1}^{*}\right)$ is the boundary of the basin of attraction to $\left(g_{1}^{i}, z_{1}^{i}\right)$.

Therefore, although the necessary conditions for the existence of a crossing trajectory exist the global conditions fail. Figures 7, 8 and 9 present three related phase diagrams illustrating this case. The three phase diagrams share several common features. First, there is a unique steady state, $\Gamma_{E, 1}$ or $\Gamma_{E}$, which is a saddle point, it is located in set $\Omega_{-}$and corresponds to a long run debtor position (because it is to the left of the $g=\bar{g}$ line). Second, the stable manifold associated to this steady state is bounded to the left by the singular surface $\mathcal{S}$ but it is also bounded to the right.

However, the phase diagrams depicted in those Figures differ in some aspects. The phase diagram in Figure 7 in addition to the steady state $\Gamma_{E, 1}$ has another steady state $\Gamma_{E, 2}$ which is locally unstable, is still within set $\Omega_{-}$and corresponds to a creditor position (because it is to the right of line $g=\tilde{g}$ ), and it has a heteroclinic trajectory connecting the two steady states. This means that either the initial values of the pre-determined variables are such that $(g(0), z(0))=\left(g_{2}^{*}, z_{2}^{*}\right)$ and the BGP with rate of growth $\gamma\left(g_{2}^{*}\right)$ is over-determinate, or $(g(0), z(0)) \in\left(\left(g_{1}^{s}, z\left(g_{1}^{s}\right)\right),\left(g_{2}^{*}, z_{2}^{*}\right)\right)$ and the DGE is determinate and converges to the BGP with rate of growth $\gamma\left(g_{1}^{*}\right)$. For other initial values DGE paths do not exist. We also depict a representative crossing trajectory, which is not a DGE path, departing from steady state $\Gamma_{E, 2}$ passing through the impasse-transversal node point $\Gamma_{I}$, entering into set $\Omega_{+}$and terminating in finite time in an attractor point belonging to set $\mathcal{S}$.

The phase diagram in Figure 8 displays only one regular steady state $\left(g_{1}^{*}, z_{1}^{*}\right)$ in set $\Omega_{-}$ and corresponding to a debtor position. In this case the higher limit for the initial value of $g$ is not given by another steady state but by a value for $g$ such that $z(0)>0$, because the stable manifold associated to the steady state is negatively sloped. Again, we depict a non-DGE crossing trajectory.

The phase diagram in Figure 9 is similar to the case in 7 with the exception that the steady state $\left(g_{1}^{*}, z_{1}^{*}\right)$ is an impasse-singular point, corresponding again to a debtor position. This steady state corresponds to a non-generic impasse-equilibrium saddle-node point. It is a singular saddle-node point because it is an unstable node from the perspective of $\Omega_{+}$and a saddle point from the perspective of $\Omega_{-}$. This is a limit case: if $\phi$ is reduced or $\bar{b}$ augmented 


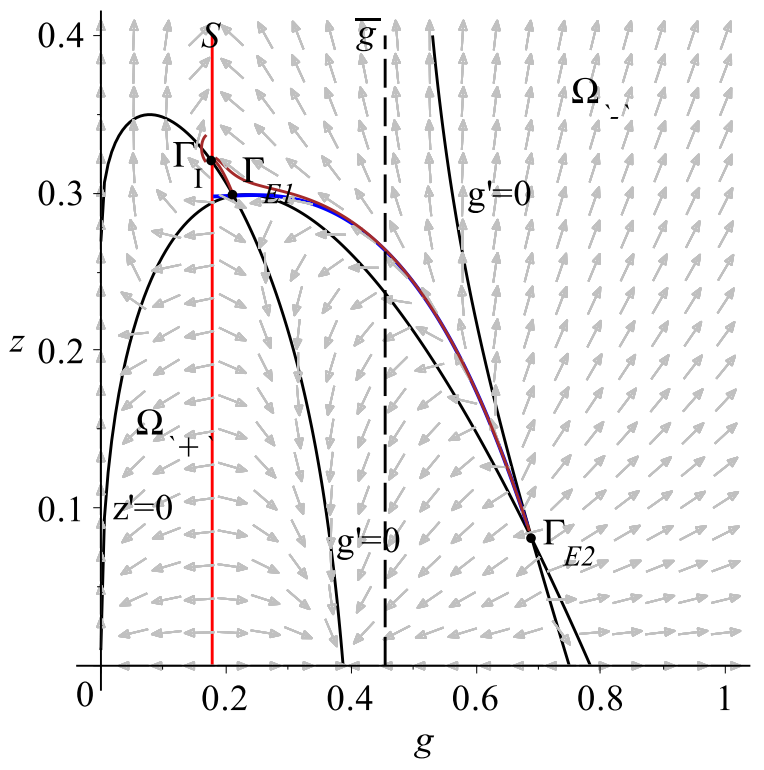

Figure 7: Phase diagram for the case L4(2) in Figure 4 and L5(7) in Figure 5, and with $\alpha=0.7, \sigma=2, \rho=0.02, \tau=0.35, \phi=0.35$ and $\bar{b}=0.3$.

then the steady state $g_{1}^{*}$ will move to the set $\Omega_{+}$, and become a regular unstable point as in phase diagram in Figure 6, or it will move to $\Omega_{-}$, and become a regular saddle-point as in phase diagram in Figure 7.

In all cases we see the equilibrium expenditure ratio can only be higher than the steady state if the government is in or close to a net creditor position.

Therefore, the following is generically verified:

Proposition 3 (Equilibrium dynamics with fiscal rules). Assume that $\bar{b} \leq \bar{b}_{s}^{*}=\bar{b}_{d}^{i}$. Then a $D G E$ path converging to an unique BGP with rate of growth $\gamma\left(g_{1}^{*}\right)$ only exists if the initial level of the ratio of the government expenditures, $g(0)$, lies between $g_{1}^{s}$ and $\min \left\{g_{2}^{*}, 1, g^{z}\right\}$, where $g^{z}$ is the point in the stable manifold such that $z=0$, and the government ratio converges to a point $g_{1}^{s} \leq g_{1}^{*}<\bar{g}$, meaning that the government is asymptotically a debtor.

It is interesting to note that for benchmark values of the parameters for the rate of time preference, intertemporal elasticity of substitution and distortionary tax rate those cases correspond to unrealistic high debt levels (above 60\%). The long run debt ratio deviates from the target $\bar{b}$ if $g^{*} \neq \tau$, which is generically the case. Observe that, one possible configuration of steady states is that $g_{1}^{*}=\tau$ and $g_{2}^{*}=\bar{g}$, which implies that $b\left(g_{2}^{*}\right)=0$. 


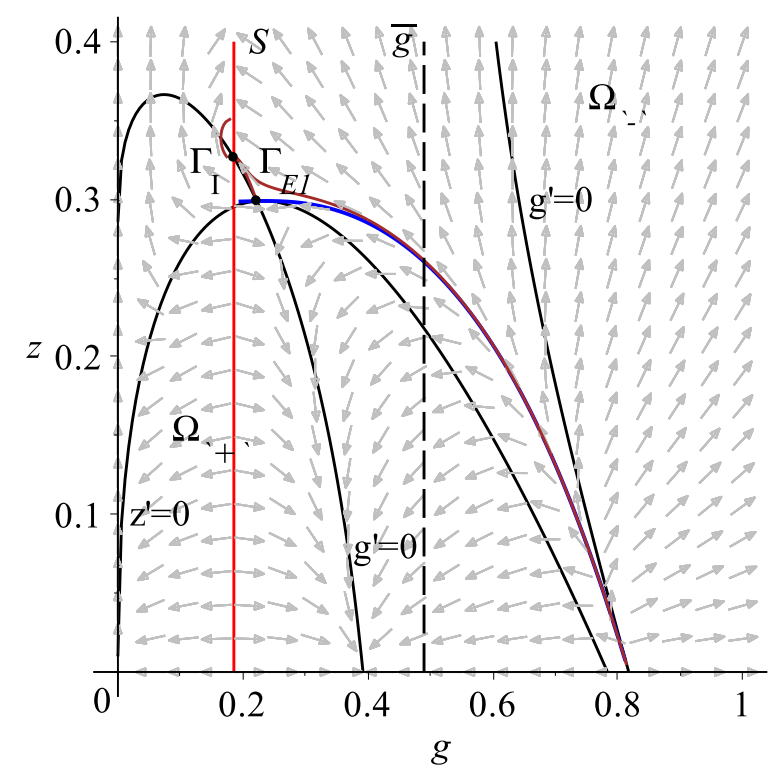

Figure 8: Phase diagram for the case $L 4(2)$ in Figure 4 and $L 5(3)$ in Figure 5, and with $\alpha=0.7, \sigma=2, \rho=0.02, \tau=0.35, \phi=0.4$ and $\bar{b}=0.35$.

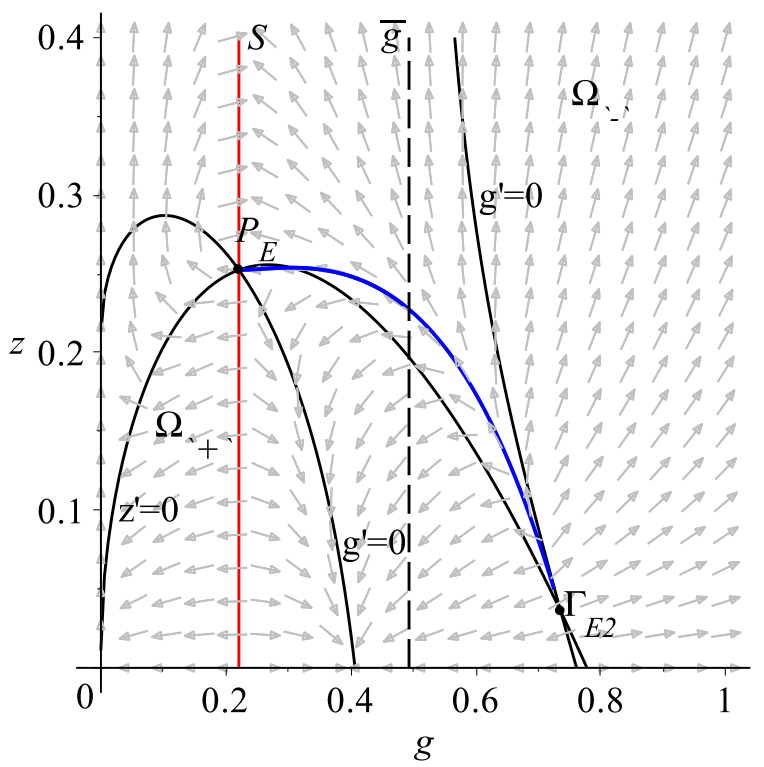

Figure 9: Phase diagram for the case L4(1) in Figure 4 and L5(8) in Figure 5, and the BGP $g^{*}=g_{1}^{*}$ is a singular steady state. We set $\alpha=0.65, \sigma=1.8, \rho=0.02, \tau=0.35, \phi=0.3$ and $\bar{b}=0.4773478078$. 


\section{Conclusion}

By introducing a realistic fiscal rule, consisting in the use of the fiscal surplus to control the difference between the actual and a target ratio of the government debt to the GDP, in an otherwise benchmark growth model with productive government expenditures, we found that depending on the design of the rule, and on the initial level of the expenditure ratio, a DGE path converging to a determinate BGP can exist or not. The existence is not guaranteed because the rule introduces impasse-singularities into the model. The types of singularities that are specifically introduced by the rule considered in this paper generate several types of dynamics which are not present in regular models: multiple over-determinate BGP's, confined determinate DGE dynamics converging to a regular or a singular BGP path. The type of dynamics can be very sensitive to small variations of the parameters defining the rule, which implies that the level of the target and the speed of the adjustment can affect the structural stability properties of the economy. 


\section{References}

Arrow, K. and Kurz, M. (1970). Public Investment, the Rate of Return and Optimal Fiscal Policy. the Johns Hopkins Press, Baltimore.

Barro, R. (1990). Government spending in a simple model of endogenous growth. Journal of Political Economy, 98:S103-S125.

Bohn, H. (1998). The behavior of U.S. public debt and deficits. Quarterly Journal of Economics, 113(3):949-963.

Bohn, H. (2008). The sustainability of fiscal policy in the united states. In Neck, R. and Sturm, J. E., editors, Sustainability of Public Debt, pages 15-49. MIT Press, Cambridge, MA.

Brito, P., Costa, L., and Dixon, H. (2017a). From Sunspots to Black Holes: singular dynamics in macroeconomic models. In Nishimura, K. and all, editors, Sunspots and Non-linear Dynamics, chapter 3. Springer.

Brito, P., Costa, L., and Dixon, H. (2017b). Singular macroeconomic dynamics and temporary indeterminacy. mimeo.

Futagami, K., Iwaisako, T., and Ohdoi, R. (2008). Debt policy rule, productive government spending, and multiple growth paths. Macroeconomic Dynamics, 12:445-462.

Ghosh, A. R., Kim, J. I., Mendoza, E. G., Ostry, J. D., and Qureshi, M. S. (2013). Fiscal fatigue, fiscal space and debt sustainability in advanced economies. Economic Journal, 123:F4-F30.

Greiner, A. (2008). Does it pay to have a balanced government budget ? Journal of Institutional and Theoretical Economics, 164(3):460-476.

Greiner, A. and Fincke, B. (2015). Public Debt, Sustainability and Economic Growth. Springer.

Irmen, A. and Kuehnel, J. (2009). Productive government expenditure and economic growth. Journal of Economic Surveys, 23:692-733.

Kamiguchi, A. and Tamai, T. (2012). Are fiscal sustainability and stable balanced growth simultaneously attainable? Metroeconomica, 63:443-457.

Kinda, T., Kolerus, C., Muthoora, P., and Weber, A. (2013). Fiscal rules at a glance. Technical report, International Monetary Fund. 
Llibre, J., Sotomayor, J., and Zhitomirskii, M. (2002). Impasse bifurcations of constrained systems. Fields Institute Communications, 31:235-256.

Nishimura, K., Seegmuller, T., and Venditti, A. (2015). Fiscal policy, debt constraint and expectation-driven volatility. Journal of Mathematical Economics, 61:305-316.

Riaza, R. (2008). Differential-Algebraic Systems: Analytical Aspects and Circuit Applications. World Scientific, Singapore.

Schmitt-Grohe, S. and Uribe, M. (1997). Balanced-budget rules, distortionary taxes, and aggregate instability. Journal of Political Economy, 105(5):976-1000.

Zhitomirskii, M. (1993). Local normal forms for constrained systems on 2-manifolds. Boletim da Sociedade Brasileira de Matemática, 24:211-232. 


\section{A Proofs}

Proof of Lemma 1 . Function (8) has the following obvious properties: $b(g)<0$ if $\min \{\bar{g}, \tilde{g}\}<$ $g<\max \{\bar{g}, \tilde{g}\}, b(g)=0$ if $g=\bar{g}, b(g)>0$ if $g<\min \{\bar{g}, \tilde{g}\}$ or $g>\max \{\bar{g}, \tilde{g}\}$, and $b(g)= \pm \infty$ if $g=\tilde{g}$. In order to determine the parameter values that map back those cases, observe that we have $\bar{g}<1$ if and only if $\bar{b}<\bar{b}_{1} \equiv(1-\alpha) / \phi$ and $\tilde{g}<1$ if and only if $\phi<\alpha \tau$. Then $\max \{\bar{g}, \tilde{g}\}<1$ if $\phi<\tau \alpha$ and $\bar{b}<\bar{b}_{1}$ and $\min \{\bar{g}, \tilde{g}\}>1$ if and only if $\phi>\tau \alpha$ and $\bar{b}>\bar{b}_{1}$ and $\min \{\bar{g}, \tilde{g}\} \leq 1 \leq \max \{\bar{g}, \tilde{g}\}$ for the other cases. The rest of the proof is obvious.

Proof of Lemma 2. Function $\delta(g)$, defined in equation (16), has the following first and second derivatives $\delta^{\prime}(g)=\tau r(g)-\phi$ and $\delta^{\prime \prime}(g)=\tau r^{\prime}(g)$. As $\delta^{\prime}(g) \gtreqless 0$ if and only if $g \lesseqgtr \tilde{g}$ and $\delta^{\prime \prime}(g)>0$ for all $g$, then it is a convex function of $g$ reaching a minimum at point $\tilde{g}$. At this point we have $\delta(\tilde{g})=\phi(1-\alpha)(\bar{g}-\tilde{g})$. Therefore $\delta(\tilde{g}) \lesseqgtr 0$ if and only if $\bar{g} \lesseqgtr \tilde{g}$. Consequently, three cases exist: (a) If $\bar{g}>\tilde{g}$ then $\delta(g)>0$ for all $g>0$ and impasse points do not exist; (b) if $\bar{g}=\tilde{g}$ then $\delta(g)=0$ if $g^{s}=\bar{g}=\tilde{g}$ and $\delta(g)>0$ otherwise, and there is a non-generic impasse point in the first case and there are no impasse points in the second; and (c) if $\bar{g}<\tilde{g}$ then there two impasse points, $g_{1}^{s}$ and $g_{2}^{s}$, can exist satisfying $0<g_{1}^{s}<\bar{g}<\tilde{g}<g_{2}^{s}$, such that $\delta(g)<0$ if $g_{1}^{s}<g<g_{2}^{s}, \delta(g)=0$ if $g=g_{1}^{s}$ or $g=g_{2}^{s}$ and $\delta(g)>0$ otherwise.

Now, consider the cases b) and c) in which impasse points exist. A necessary condition for this is $\bar{g} \leq \tilde{g}$. We need to determine under which conditions the impasse points are admissible, i.e., belong to the domain $(0,1)$. If $\bar{g}=\tilde{g}$ then the singular point satisfies $g_{s}<1$ if and only if $\delta(1)<0$ and $\delta^{\prime}(1)>0$, that is if $\phi<\tau \alpha$ and $\bar{b}<\bar{b}_{1}=(1-\tau) /(\alpha \tau)$. If $\bar{g}<\tilde{g}$ then two cases are possible: (a) there are two admissible singular points satisfying $g_{1}^{s}<g_{2}^{s}<1$ only if $\delta(1)>0$ and $\delta^{\prime}(1)>0$; (b) there is only one admissible singular point satisfying $g_{1}^{s}<1$ if $\delta(1)<0$. However, $\delta(1) \lesseqgtr 0$ if and only if $\bar{b} \lesseqgtr \bar{b}_{1}$ and $\delta^{\prime}(1) \lesseqgtr 0$ if and only if $\phi \gtreqless \alpha \tau$ which is equivalent to $\tilde{g} \gtreqless 1$.

Proof of Lemma 3. Impasse-transversal points, $\left(g^{i}, z^{i}\right)$, are determined by solving $\delta(g)=0$ and $\psi(g, z)=0$ within set $\Omega$, i.e., for $g \in(0,1)$ and $z>0$. First, from Lemma 2 we already know that a condition for existence of impasse-points $g^{s}$ such that $\delta(g)=0$ is $\bar{g} \leq \tilde{g}$. As impasse points verify $\operatorname{\tau r}\left(g^{s}\right) \neq \phi$ and $g^{s} \neq \bar{g}$ then $\psi(g, z)=0$ if and only if $z^{i}=\zeta\left(g^{s}\right)$. Then, taking stock of Lemma 2 which determines the conditions for the existence $\left(\bar{b} \leq \bar{b}_{s}\right)$ of one $\left(\phi>\alpha \tau\right.$ and $\left.\bar{b}<\bar{b}_{1}^{s}\right)$ or two impasse points $\left(\phi>\alpha \tau\right.$ and $\left.\bar{b}^{s}>\bar{b}>\bar{b}_{1}^{s}\right)$ we have only to check if $\zeta\left(g^{s}\right)>0$. Solving jointly the system $\delta(g, \bar{b})=0$ and $\zeta(g, \bar{b})=0$ we find, implicitly, two critical values $\bar{b}_{i}$, such that $\bar{b}_{i}=\{\bar{b}: \delta(g, \bar{b})=0, Z(g, \bar{b})=0\}$, where

$$
Z(g) \equiv g^{2}-\left((1-\alpha)(1+\bar{g})+\tau^{2}\right) g+(1-\alpha)\left(\bar{g}(1-\alpha(1-\tau))-\alpha \tau^{2}\right) .
$$

This is because $\zeta(g) \geq 0$ if and only if $Z(g, \bar{b}) \geq 0$. Two image singular points exist if, additionally, $\bar{b}^{s}>\bar{b}>\bar{b}^{i}$ and one impasse-transversal point exists if $\bar{b}<\bar{b}_{i}$. Comparing the values of those critical levels for $\bar{b}$, we find that there are five possible cases, when there 
exists at least one singular point: two impasse surfaces, $\mathcal{S}_{1}$ and $\mathcal{S}_{2}$, one transversal point in both surfaces, one transversal point in surface $\mathcal{S}_{1}$ and none in $\mathcal{S}_{2}$, or one surface $\mathcal{S}=\mathcal{S}_{1}$ and one or zero transversal points, or zero transversal points

In order to determine the partition in impasse surfaces that are introduced by transversal points, we should observe that attractor (repeller) points verify $\delta^{\prime}(g) \psi(g, z)<0\left(\delta^{\prime}(g) \psi(g, z)>\right.$ $0)$. As $\delta^{\prime}\left(g_{1}^{s}\right)<0$ and $\delta^{\prime}\left(g_{2}^{s}\right)>0$ and because for any singular point $\left(\tau r\left(g_{s}\right)-\phi\right)\left(g_{s}-\bar{g}\right)>0$ then $\operatorname{sign}\left(\psi\left(g_{s}, z\right)\right)=\operatorname{sign}\left(z-\zeta\left(g_{s}\right)\right)$ then the singular point $g_{1}^{s}$ is an attractor (repeller) if $z>z_{1}^{i}=\zeta\left(g_{1}^{s}\right)\left(z<z_{1}^{i}=\zeta\left(g_{1}^{s}\right)\right)$ and the singular point $g_{2}^{s}$ is an attractor (repeller) if $z<z_{2}^{i}=\zeta\left(g_{2}^{s}\right)\left(z>z_{2}^{i}=\zeta\left(g_{2}^{s}\right)\right)$.

Proof of Lemma 4. The Jacobian of system (25)-(26), evaluated at an impasse-transversal point $\left(g^{i}, z^{i}\right)$, is

$$
J\left(g^{i}, z^{i}\right)=\left(\begin{array}{cc}
g^{i} \psi_{g}^{\prime}\left(g^{i}, z^{i}\right) & g^{i} \psi_{z}^{\prime}\left(g^{i}, z^{i}\right) \\
z^{i} \delta^{\prime}\left(g^{i}\right)\left(z^{i}-z\left(g^{i}\right)\right) & 0
\end{array}\right),
$$

where

$$
\begin{aligned}
& \psi_{z}^{\prime}\left(g^{i}, z^{i}\right)=\alpha \tau\left(g^{i}-\bar{g}\right)\left(\tau r\left(g^{i}\right)-\phi\right) \\
& \psi_{g}^{\prime}\left(g^{i}, z^{i}\right)=-\zeta^{\prime}\left(g^{i}\right) \psi_{z}^{\prime}\left(g^{i}, z^{i}\right) .
\end{aligned}
$$

As $z^{i}=\zeta\left(g^{i}\right)$ their algebraic properties depends only on $g^{i}$. Therefore, its trace, determinant and discriminant are

$$
\begin{aligned}
\operatorname{tr}\left(J^{i}\right) & =-g^{i} \zeta^{\prime}\left(g^{i}\right) \psi_{z}^{\prime}\left(g^{i}, \zeta\left(g^{i}\right)\right) \\
\operatorname{det}\left(J^{i}\right) & =g^{i} z^{i} \delta^{\prime}\left(g^{i}\right)\left(\zeta\left(g^{i}\right)-z\left(g^{i}\right)\right) \psi_{z}^{\prime}\left(g^{i}, \zeta\left(g^{i}\right)\right) \\
\Delta\left(J^{i}\right) & =g^{i} \psi^{\prime}\left(g^{i}\right)\left(g^{i}\left(\zeta^{\prime}\left(g^{i}\right)\right)^{2}+4 \zeta\left(g^{i}\right)\left(\zeta\left(g^{i}\right)-z\left(g^{i}\right)\right)\right) .
\end{aligned}
$$

We have $\psi_{z}^{\prime}\left(g^{i}\right)=\psi_{z}^{\prime}\left(g^{i}, \zeta\left(g^{i}\right)\right)>0$ for any $g^{i}$. Therefore, $\operatorname{sign}\left(\operatorname{tr}\left(J^{i}\right)\right) \neq \operatorname{sign}\left(\zeta^{\prime}\left(g^{i}\right)\right)$ and $\operatorname{sign}\left(\operatorname{det}\left(J^{i}\right)\right)=\operatorname{sign}\left(\delta^{\prime}\left(g^{i}\right)\left(\zeta\left(g^{i}\right)-z\left(g^{i}\right)\right)\right)$. The discriminant is positive if $z^{i}=\zeta\left(g^{i}\right) \leq z\left(g^{i}\right)$ and may be zero or negative only if $\zeta\left(g^{i}\right)<z\left(g^{i}\right)$.

After some algebra we get the determinant of Jacobian $J\left(g^{i}\right) \equiv J\left(g^{i}, \zeta\left(g^{i}\right)\right)$

$$
\operatorname{det} J\left(g^{i}\right)=\alpha z^{i} g^{i}\left(\tau r\left(g^{i}\right)-\phi\right)^{2} D\left(g^{i}\right)\left(g^{i}-\bar{g}\right)\left(g^{i}-g_{d}^{i}\right)
$$

where

$$
D\left(g^{i}\right) \equiv \frac{\rho \alpha \tau+\phi(\sigma(1-\alpha \tau)-(1-\tau))}{\alpha \tau \sigma g^{i}}
$$

and $g_{d}^{i}$ is in equation (27). If $\sigma>1$ then $D\left(g^{i}\right)>0$ and $g_{d}^{i}<\bar{g}$. This implies, because we have proved that $g_{1}^{i}<\bar{g}<g_{2}^{i}$, $\operatorname{det}\left(J\left(g_{2}^{i}\right)\right)>0$ and $\operatorname{det}\left(J\left(g_{1}^{i}\right)\right)>0$ if $g_{1}^{i}<g_{d}^{i}<\bar{g}$ and $\operatorname{det}\left(J\left(g_{1}^{i}\right)\right) \leq 0$ if $g_{1}^{i} \leq g_{d}^{i}<\bar{g}$. Therefore $\left(g_{1}^{i}, z_{1}^{i}\right)$ can be a transversal saddle, node or focus and $\left(g_{2}^{i}, z_{2}^{i}\right)$ can only be a node or a focus. In order to discriminate between the two 
cases, we need to determine the sign of the discriminant of the Jacobian evaluated at every impasse-transversal point.

The sign of the discriminant $\Delta\left(J^{i}\right)$ depends on a quadratic polynomial in $(1 / g)$. This means that there can be generically, zero or two critical values for $g^{i}$, say $g_{\Delta, 1}^{i}<g_{\Delta, 2}^{i}$. In the first case the two image-transversal points are nodes and in the second one they are foci. Evaluating the trace for impasse-transversal nodes is required for determining the direction of the crossing trajectories. If the trace is negative (positive) then there is crossing from the adjacent $\Omega_{+}$set to set $\Omega_{-}$(from $\Omega_{-}$to the adjacent $\Omega_{+}$set). We readily see that $\operatorname{sign}\left(\operatorname{tr}\left(J^{i}\right)\right)=-\operatorname{sign}\left(\zeta^{\prime}\left(g^{i}\right)\right)$, where the sign of $\zeta^{\prime}\left(g_{1}^{i}\right)$ is ambiguous but $\zeta^{\prime}\left(g_{2}^{i}\right)>0$. That is, when there is an impasse-transversal node at $\left(g_{2}^{i}, z_{2}^{i}\right)$ there is crossing from $\Omega_{-}$to $\Omega_{+, 2}$.

If $g_{i}^{*}=\bar{g}=\tilde{g}$ then the two impasse-transversal points collapse to one and $\operatorname{as} \operatorname{tr}\left(J_{i}^{*}\right)=$ $\operatorname{det}\left(J_{i}^{*}\right)=0$ we have a singularity induced bifurcation point with co-dimension 2 .

Proof of Lemma 5. Equilibrium steady states values $\left(g^{*}, z^{*}\right)$ must verify three conditions: first, they are fixed points of the system (20)-(21); second, they belong to set $\Omega$; third, the transversality condition should be satisfied. A regular (singular) steady state additionally verifies $\delta(g) \neq 0(\delta(g)=0)$.

First, we determine the fixed points of system (20)-(21) in the $\Omega$ domain. From equation (21), we find $\dot{z}=0$ if and only if $z=0$ or $z^{*}=z\left(g^{*}\right)$. The first point is not in set $\Omega$ and the second is monotonously related to $g^{*}$ and is a BGP if $z^{*}>0$. On the other hand the set of fixed points for $g$ is $g^{*}=\{g \in(0,1): \Psi(g) \equiv \psi(g, z(g))=0\}$ where

$$
\Psi(g)=\alpha(\tau r(g)-\phi)\left(\varphi_{d}(g) \tau r(g)-\varphi_{n}(g)\right) / \sigma
$$

and $\varphi_{d}(g) \equiv\left((\tau \sigma+(1-\tau)(\sigma-1)) g-\left(\tau^{2} \sigma+\bar{g}(1-\tau)(\sigma-1)\right)\right) / \tau$, and $\varphi_{n}(g) \equiv(\sigma \phi-$ $\rho) g-(\sigma \phi \tau-\rho \bar{g})$. It is useful to define $\varphi(g)=\varphi_{n}(g) / \varphi_{d}(g)$, that is

$$
\varphi(g)=\frac{\tau(\sigma \phi-\rho)}{\tau \sigma+(1-\tau)(\sigma-1)}\left(\frac{g-g_{1}}{g-g_{0}}\right)
$$

where

$$
g_{0} \equiv \frac{\tau^{2} \sigma+\bar{g}(1-\tau)(\sigma-1)}{\tau \sigma+(1-\tau)(\sigma-1)}, \text { and } g_{1} \equiv \frac{\phi \sigma \tau-\rho \bar{g}}{\sigma \phi-\rho} .
$$

The fixed points of $\Psi(g)=0$ are determined from two conditions: $\operatorname{\tau r}(g)=\phi$ and $\operatorname{\tau r}(g)=\varphi(g)$. Then one fixed point is always $g_{1}^{*}=\tilde{g}$ (solving equation $\operatorname{\tau r}(g)=\phi$ ). Other fixed points are the solutions of equation $\operatorname{\tau r}(g)=\varphi(g)$. We find three cases: (a) if $\sigma \phi<\rho$ and $g_{1}>g_{0}>0$ then there is a second fixed point $g_{2}^{*} \in\left(g_{0}, g_{1}\right)$; (b) if $\sigma \phi>\rho$ and $g_{0}>0 \geq g_{1}$ then there is a second fixed point $g_{2}^{*}>g_{0}$; and (c) if $\sigma \phi>\rho$ and $g_{0}>g_{1}>0$ then there are two more fixed points, $g_{2}^{*}$ and $g_{3}^{*}$, such that $g_{2}^{*}<g_{1}<g_{0}<g_{3}^{*}$. But, as $g_{1}=0$ if and only if $\bar{b}=\bar{b}_{1}^{*}$, in equation (28), then the first two cases can be taken together if $\bar{b}<\bar{b}_{1}^{*}$. 
Next, we have to check if the transversality condition holds for all those roots of equation $\Psi(g)=0$. First, the rates of growth for consumption, capital stock and the government debt are derived in equation (7), (17) and (12). Using the definitions in equation (19) we find $\gamma_{K}(g, z)-\gamma(g)=z(g)-z$. Then $\gamma_{K}^{*}=\gamma_{K}\left(g^{*}, z^{*}\right)=\gamma^{*}=\gamma\left(g^{*}\right)$ along any fixed point of the system (20)-(21).

On the other hand

$$
\gamma_{B}(g)-\gamma(g)=\frac{\varphi_{d}(g)}{\sigma(g-\bar{g})}(\tau r(g)-\varphi(g))
$$

Then $\gamma_{B}(g)-\gamma(g)=0$ if and only if: (a) $\varphi_{d}(g) \neq 0$ and $\tau r(g)=\varphi(g)$; or (b) $g=\bar{g}$ and $\operatorname{\tau r}(g)=\varphi(g)=\phi$, that is if $\bar{g}=\tilde{g}$. Observe that if $\tau r(g)=\phi$ and $\bar{g} \neq \tilde{g}$ then $\gamma_{B}(g)-\gamma(g) \neq 0$. Therefore: (1) a fixed point such that $z^{*}=z\left(g^{*}\right)$ and $\tau r\left(g^{*}\right)=\varphi\left(g^{*}\right)$ implies $\gamma_{B}^{*}=\gamma_{K}^{*}=\gamma^{*}=\left((1-\tau) r\left(g^{*}\right)-\rho\right) / \sigma$, and, then $\rho+\sigma \gamma^{*}-\gamma_{K}^{*}=\rho+\sigma \gamma^{*}-\gamma_{B}^{*}=$ $\rho+(\sigma-1) \gamma^{*}=\left(\rho+(\sigma-1)(1-\tau) r\left(g^{*}\right)\right) / \sigma>0$ if $\sigma \geq 1$, and the transversality condition holds; (2) a fixed point such that $z^{*}=z\left(g^{*}\right)$ and $\tau r\left(g^{*}\right)=\phi$ leads to two possible cases: if $g^{*}=\bar{g}$ then $g^{*}=\tilde{g}=\bar{g}$ implying $\gamma_{B}^{*}=\gamma_{K}^{*}=\gamma^{*}=\left((1-\tau) r\left(g^{*}\right)-\rho\right) / \sigma$ and the transversality condition is also satisfied; however, if $g^{*} \neq \bar{g}$ then $\gamma_{B}^{*}=(1-\tau) \phi / \tau$ which is different from $\gamma_{K}^{*}=\gamma^{*}=\left((1-\tau) r\left(g^{*}\right)-\rho\right) / \sigma$, and because $\rho+\sigma \gamma^{*}-\gamma_{B}^{*}=\rho+(1-\tau) \phi / \tau-\rho-(1-\tau) \phi / \tau=0$ the transversality condition is not satisfied.

At last we check if the fixed points verifying the transversality condition belong to the domain $\Omega$ : that is whether $g^{*} \in(0,1)$ and $z\left(g^{*}\right)>0$. As $z(0)=\rho / \sigma>0$ and, if we assume that $\rho<\alpha(1-\tau)), z(1)=(\rho-\alpha(1-\tau)) / \sigma<0$ and $z^{\prime}(0)>0$ and $z^{\prime}(1)<0$ then $g^{*} \in(0,1)$ if $z\left(g^{*}\right)>0$. As $z(g)>0$ if and only if $(\sigma(1-g) / \alpha-(1-\tau)) r(g)+\sigma \rho>0$ then there is a unique critical level $g_{z}$ such that $z(g)>0$ if and only if $g<g_{z}$. Then by substituting in the equilibrium condition we find a critical value for a parameter $\bar{b}, \bar{b}_{z}^{*}$, such that $\varphi\left(\bar{b}_{z}^{*}, g_{z}\right)=\operatorname{\tau r}\left(g_{z}\right)$. This value exists and is unique and we have $z\left(g^{*}\right)>0$ if and only if $\bar{b}<\bar{b}_{z}^{*}$.

Now, we address the location of the fixed points, as regards the impasse surface(s). Taking $\operatorname{\tau r}(g)=\varphi(g)$ and solving jointly with $\delta(g)=0$ we obtain both singular fixed points and the constraint in the parameters for their existence. Factoring the expression $\operatorname{\tau r}(g)>0$ in both conditions, we get $\tau r(g)=\frac{\phi(g-(1-\alpha) \bar{g})}{\alpha g}=\varphi(g)$ that is equivalent to $\phi(g-(1-\alpha) \bar{g}) \varphi_{d}(g)=$ $\alpha g \varphi_{n}(g)$ and $g_{s}^{*}$. However only the last one can be an equilibrium BGP. Observe that, when there are two BGP's only the one corresponding to the smaller $g$ can be a singular BGP. From $\delta\left(g_{s}^{*}\right)=0$ we get the critical value $\bar{b}_{s}^{*}$.

If we consider additionally the proofs of Lemmas 1, 2, and the constraint $z(g)>0$ (and the fact that if $z(1)<0$ then $\operatorname{\tau r}(1)>\psi(1)$, and if $z(1)<0$ then $\bar{g}<1$ ), we have the following cases: (i) if $\mathrm{f} \bar{b}>\bar{b}_{1}^{*}$ then there is a unique BGP and $\delta\left(g^{*}\right)>0$ and $\tilde{g}<g^{*}<\bar{g}<1$; (ii) if $\bar{b} \geq \bar{b}_{z}$ then there are two roots of $\Psi(g)=0$ but only one, $g_{1}^{*}$, verifies $z(g)>0$. In addition we always have $g_{1}^{*}<\min \{\bar{g}, \tilde{g}\}$ and the $\operatorname{sign}$ of $\delta\left(g_{1}^{*}\right)$ is positive (negative) if $\bar{b}>(<) \bar{b}_{s}^{*}$; (iii) if $\bar{b}=\bar{b}_{s}^{*}$ then $\delta\left(g_{1}^{*}\right)=0$; (iv) if $\bar{b}<\min \left\{\bar{b}_{z}, \bar{b}_{1}^{*}\right\}$ then there are two roots of $\Psi(g)=0$, 
verifying the transversality condition and $z(g)>0$. In addition the following relationship is verified $\min \left\{g_{1}^{*}, g_{1}^{s}\right\} \leq \max \left\{g_{1}^{*}, g_{1}^{s}\right\}<\bar{g}<g_{2}^{*}<\tilde{g}<g_{2}^{s}$. But $g_{1}^{*}=\min \left\{g_{1}^{*}, g_{1}^{s}\right\}$ if $\bar{b}>\bar{b}_{s}^{*}$, $g_{1}^{s}=\min \left\{g_{1}^{*}, g_{1}^{s}\right\}$ if $\bar{b}<\bar{b}_{s}^{*}$ and $\left.g_{1}^{*}=g_{1}^{s}\right\}$ if $\bar{b}=\bar{b}_{s}^{*}$. The creditor position associated to the steady states is easily determined from the previous inequalities and from Lemma 1.

Proof of Lemma 6. The Jacobian of system (20)-(21) evaluated at a regular BGP $\left(g^{*}, z^{*}\right)$ is

$$
J^{*} \equiv J\left(g^{*}, z^{*}\right)=\frac{1}{\delta\left(g^{*}\right)}\left(\begin{array}{cc}
g^{*} \psi_{g}^{\prime}\left(g^{*}, z^{*}\right) & g^{*} \psi_{z}^{\prime}\left(g^{*}, z^{*}\right) \\
-z^{*} z^{\prime}\left(g^{*}\right) \delta\left(g^{*}\right) & -z^{*} \delta\left(g^{*}\right)
\end{array}\right) .
$$

It has trace and determinant

$$
\begin{aligned}
\operatorname{tr}\left(J^{*}\right) & =\frac{z^{*} \delta\left(g^{*}\right)+g^{*} \psi_{g}^{\prime}\left(g^{*}, z^{*}\right)}{\delta\left(g^{*}\right)} \\
\operatorname{det}\left(J^{*}\right) & =\frac{z^{*} g^{*}}{\delta\left(g^{*}\right)}\left(\psi_{g}^{\prime}\left(g^{*}, z^{*}\right)+\psi_{z}^{\prime}\left(g^{*}, z^{*}\right) z^{\prime}\left(g^{*}\right)\right)
\end{aligned}
$$

However, because $\Psi^{\prime}\left(g^{*}\right)=\psi_{g}^{\prime}\left(g^{*}, z^{*}\right)+\psi_{z}^{\prime}\left(g^{*}, z^{*}\right) z^{\prime}\left(g^{*}\right)$ (see proof of Lemma 5 ) then the trace and the determinant become, equivalently

$$
\begin{aligned}
\operatorname{tr}\left(J^{*}\right) & =z^{*}+g^{*}\left(\frac{\Psi^{\prime}\left(g^{*}\right)}{\delta\left(g^{*}\right)}-\frac{\psi_{z}^{\prime}\left(g^{*}, z^{*}\right) z^{\prime}\left(g^{*}\right)}{\delta\left(g^{*}\right)}\right) \\
\operatorname{det}\left(J^{*}\right) & =z^{*} g^{*} \frac{\Psi^{\prime}\left(g^{*}\right)}{\delta\left(g^{*}\right)}
\end{aligned}
$$

If $\bar{g}>\tilde{g}$ as $\delta\left(g^{*}\right)>0$ and $\Psi^{\prime}\left(g^{*}\right)>0$ for any root of $\Psi(g)=0$, with the exception of $g^{*}=\tilde{g}$, then all the equilibrium BGP are locally unstable nodes or foci, i.e. the dimension of the local stable manifold is zero. If $\bar{g}<\tilde{g}$ the two fixed points of $\Psi(g)=0$, with the exception of $g^{*}=\tilde{g}$ always verify $\Psi^{\prime}\left(g_{1}^{*}\right)>0$ and $\Psi^{\prime}\left(g_{2}^{*}\right)<0$. In addition as $g_{1}^{s}<\bar{g}<g_{2}^{*}$ then we always have $\delta\left(g_{2}^{*}\right)<0$ and therefore the point $g_{2}^{*}$ is also always an unstable node or focus. On the other hand, if $g_{1}^{s}<g_{1}^{*}\left(g_{1}^{s}>g_{1}^{*}\right)$ then $\delta\left(g_{1}^{*}\right)<0$ and $g_{1}^{*}$ is a saddle point (unstable node or focus). 